\title{
Bubble propagation in Hele-Shaw channels with centred constrictions
}

\author{
Andrés Franco-Gómez ${ }^{1}$, Alice B. Thompson ${ }^{2}$, Andrew L. Hazel ${ }^{2}$ and \\ Anne Juel ${ }^{1}$ 田 \\ ${ }^{1}$ Manchester Centre for Nonlinear Dynamics \& School of Physics \& Astronomy, The \\ University of Manchester, Manchester M13 9PL, UK. \\ ${ }^{2}$ Manchester Centre for Nonlinear Dynamics \& School of Mathematics, The University of \\ Manchester, Manchester M13 9PL, UK.
}

6 December 2017

\begin{abstract}
We study the propagation of finite bubbles in a Hele-Shaw channel, where a centred occlusion (termed a rail) is introduced to provide a small axially-uniform depth constriction. For bubbles wide enough to span the channel, the system's behaviour is similar to that of semi-infinite fingers and a symmetric static solution is stable. Here, we focus on smaller bubbles, in which case the symmetric static solution is unstable and the static bubble is displaced towards one of the deeper regions of the channel on either side of the rail. Using a combination of experiments and numerical simulations of a depth-averaged model, we show that a bubble propagating axially due to a small imposed flow rate can be stabilised in a steady symmetric mode centred on the rail through a subtle interaction between stabilising viscous forces and destabilising surface tension forces. However, for sufficiently large capillary numbers $\mathrm{Ca}$, the ratio of viscous to surface tension forces, viscous forces in turn become destabilising thus returning the bubble to an off-centred propagation regime. With decreasing bubble size, the range of $\mathrm{Ca}$ for which steady centred propagation is stable decreases, and eventually vanishes through the coalescence of two supercritical pitchfork bifurcations. The depth-averaged model is found to accurately predict all the steady modes of propagation observed experimentally, and provides a comprehensive picture of the underlying steady bifurcation structure. However, for sufficiently large imposed flow rates, we find that initially centred bubbles do not converge onto a steady mode of propagation. Instead they transiently explore weakly unstable steady modes, an evolution which results in their break-up and eventual settling into a steady propagating state of changed topology.
\end{abstract}

\section{Introduction}

Understanding the motion of gas bubbles within liquid-filled vessels is a fundamental problem in fluid mechanics, and the interaction between the bounding geometry and deformable

$\ddagger$ Corresponding author: anne.juel@manchester.ac.uk 
gas-bubble can provoke non-trivial dynamics that is quite different from that of bubbles in unbounded flow [1]. Pure hydrodynamic interactions induce migration of the bubbles normal to the predominant direction of flow and they accumulate at particular locations within the vessel's cross-section [2]. Although our principal motivation for this study is fundamental fluid-mechanical interest, a detailed understanding of these effects can be used to develop passive bubble sorting applications in lab-on-a-chip devices [3]. Migratory mechanisms also operate on particles and droplets and the resulting applications in microfluidics include assays for chemical and biological dynamics [4], segregation of blood components [5], trapping of micro-organisms in water [6], and formation and purification of emulsions or colloids [7].

Passive bubble sorting requires bubbles of different properties to be transported at different positions within the vessel's cross-section. It is then straightforward to use geometric separators to collect bubbles with the required properties. The accuracy and sensitivity of these methods will both be maximised if the cross-sectional position of a bubble can be significantly altered in response to small changes in governing parameters: a situation that arises naturally if the positional migration is a consequence of changes in the stability and number of bubble propagation modes, i.e. near bifurcation points of the underlying dynamical system. Thus, nonlinearity of the system is essential for such sorting devices. For sufficiently small vessels, inertial effects in the liquid are often negligible and the nonlinear effects arise only through the possible deformation of the gas-liquid interface.

If there are no inertial effects in the fluid then rigid particles do not migrate within the cross section [8], whereas the deformability of droplets and bubbles allows them to migrate [2, 9] through a subtle interplay between surface tension and viscous forces, whose relative importance can be quantified by a capillary number, $C a=\mu^{*} U_{b}^{*} / \sigma^{*}$, where $\mu^{*}$ is the viscosity of the suspending fluid, $U_{b}^{*}$ is the bubble velocity and $\sigma^{*}$ is the surface tension at the interface between the suspending liquid and gas bubble. For pressure-driven flow in vessels with circular, annular or rectangular cross-sections, a deformable object will always migrate towards the centre of the cross-section [10,11]. Thus, the system must be modified in some fashion in order to induce multiple propagation modes and hence to allow robust passive sorting.

In the absence of other external forcing, the only possible modifications are changes to the vessel geometry. For example, Abbyad et al. [12] showed that drops may be guided to desired locations in a microchannel by inscribing grooves in its top surface. These grooves anchor the droplets by enabling them to expand to reduce their surface energy and the grooves thus passively guide the droplets along pre-determined paths for moderate flow rates. Furthermore, multiple grooves of different widths have been used to sort droplets by their size or capillary number [13]. Although such grooves must be specifically manufactured and hence are typically used as passive control mechanisms, droplets can also be actively moved between grooves by use of cheap commercially available DVD lasers [14].

In this paper, we consider the propagation of finite gas bubbles suspended within silicone 
oil driven through a vessel of large-aspect-ratio rectangular cross-section, a Hele-Shaw channel, that we modify by the addition of a centred localised height constriction, henceforth termed a "rail". Two-phase flow in Hele-Shaw channels in the absence of such rails has been extensively studied since the seminal papers of Saffman \& Taylor [15, 16]. In these systems, depth-averaged models predict a variety of possible bubble propagation modes [17, 18], but for non-zero surface tension only one, centred, propagation mode is stable [11].

Previous studies of two-phase displacement flows in channels constricted by a rail have focused on the propagation of open bubbles, often referred to as fingers, in channels of both small [19,-21] and large [22, 23] cross-sectional aspect ratios, and closed bubbles longer than the width of the channel [24]. These studies have uncovered a wide variety of steady and oscillatory propagation modes: at low flow rates surface tension dominates and a finger will propagate centrally along the channel; at high flow rates the fingers tend to propagate asymmetrically along one side of the rail, which avoids displacing fluid within the constricted region in which the local viscous resistance is greatest. For intermediate flow rates a variety of centred and asymmetric oscillatory solutions have been observed.

In an unoccluded channel, bubbles with finite volume have a number of features in common with air fingers; multiple solution branches exist, but only a single, centred branch is stable. Franco-Gómez et al. [25] recently studied the behaviour of small bubbles in the presence of a rail with width comparable to the bubble diameter and found that these bubbles can exhibit bistability of centred and off-centred propagation modes inside a tongue-shaped region of the parameter space spanning bubble size and imposed flow rate; this bistability can in principle be used as a mechanism for passive sorting of bubbles by size. These small bubbles remain nearly circular even during migration across the channel.

For the present work, we consider the transition in behaviour as bubble size varies from diameters comparable to the rail width to diameters comparable to the channel width, which bridges the two recent studies of Franco-Gómez et al. [23, 25]. We shall concentrate on the fundamental questions of how the number and stability of different propagation modes vary with changes in flow rate and bubble volume for a given rail geometry. We find that viscous forces can act to either stabilise or destablise bubbles propagating in a centred configuration over the rail depending on the value of $C a$ and the size of the bubble. For large flow rates, we find that an initially centred bubble selects neither a steady symmetric nor steady asymmetric mode of propagation, but exhibits complex unsteady behaviour, with significant shape deformation, that appears to be organised by unstable steadily propagating solutions of the system. Unstable invariant solutions have recently been recognised as playing a key role in the transition to turbulence in shear flows [26, 27] and we conjecture that they may play a similar role in the transition to the disordered behaviour that we have observed in this system.

The paper is structured as follows. The experimental and numerical methods are presented in sections 2 and 3, respectively. The steady modes of propagation of a finite bubble are discussed in section 4.1 through the comparison between experiment and numerical 
simulation. We find that a centred mode of propagation disappears with decreasing bubble size, which is presented in section 4.2. The bifurcation structure underlying steady bubble propagation is established through numerical simulations in section 4.3 and used to interpret the unsteady propagation of bubbles at large flow rates in section 4.4 . Conclusions are given in section 5 ,

\section{Experimental methods}

(a)

\begin{tabular}{|c|c|c|}
\hline glass plate & $W^{*}$ & \\
\hline wall $H^{*}$ & $h^{*} w^{*}$ & wall \\
\hline glass plate & thin rail & \\
\hline
\end{tabular}

(b) oil reservoir

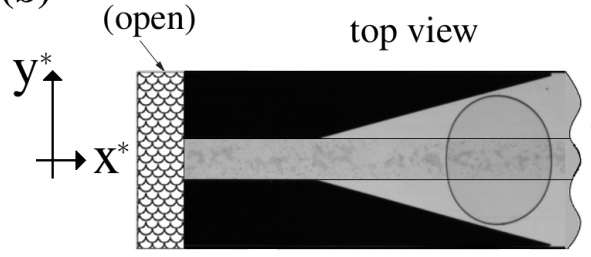

oil reservoir (sealed)

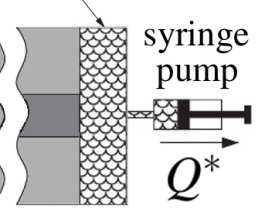

Figure 1. Schematic diagram of the experimental system. (a) Cross-sectional view of the channel (width $W^{*}=30.0 \mathrm{~mm}$ and height $H^{*}=1.0 \mathrm{~mm}$ ) with a prescribed depth variation: an axially uniform, thin rail (width $w^{*}=7.0 \mathrm{~mm}$ and height $h^{*}=24.0$ $\mu \mathrm{m}$ ) is positioned on the bottom boundary, symmetrically about the centreline of the channel. (b) Top view of the experimental system, showing the constricted channel upstream of the flow channel used to position the bubble in a centred static initial configuration.

A schematic diagram of the experimental setup is shown in Figure 11. It is described in detail in previous work [23, 25], and thus, we only provide a brief description of the points pertinent to the present study. All dimensional quantities are starred. The experimental channel consisted of two parallel float glass plates of dimensions $60 \mathrm{~cm} \times 10 \mathrm{~cm} \times 2 \mathrm{~cm}$ separated by brass sheets of uniform height $H^{*}=1.0 \mathrm{~mm}$, accurate to within $0.1 \%$ (Figure 17). The width of the channel was $W^{*}=30.0 \pm 0.1 \mathrm{~mm}$, so that its aspect ratio was $\alpha=W^{*} / H^{*}=30$ (with the exception of section 4.4, where we also consider a channel with $W^{*}=40 \mathrm{~mm}$ so that $\alpha=40$ ). A thin rail of uniform width $w^{*}=7.0 \pm 0.1 \mathrm{~mm}$ (and also $w^{*}=10.0 \pm 0.1 \mathrm{~mm}$ in section 4.4), and thickness $h^{*}=24 \pm 1 \mu \mathrm{m}$ (2.4\% of the height of the cell) was cut from a sheet of polypropylene film, and bonded to the bottom plate of the channel with its centreline aligned along the centreline of the channel. Measurements of the rail profile using a step profilometer (DekTak II) are presented in the supplementary material of [25]. The widths of the two regions between the edge of the rail and the side walls of the channel were carefully calibrated to differ by less than $1 \%(<100 \mu \mathrm{m})$, and the channel was levelled horizontally to better than $0.05^{\circ}$.

Perspex fluid reservoirs with a small bleed hole on their upper surface were attached to both ends of the channel, with a tight seal achieved by using rubber gaskets (Figure $1 \mathrm{p}$ ). The 
channel was completely filled with silicone oil (Basildon Chemicals Ltd), which had viscosity $\mu^{*}=1.0 \times 10^{-2} \mathrm{~Pa} \mathrm{~s}$, density $\rho^{*}=961 \mathrm{~kg} \mathrm{~m}^{-3}$ and surface tension $\sigma^{*}=2.1 \times 10^{-2} \mathrm{~N} \mathrm{~m}^{-1}$ at the laboratory temperature of $21^{\circ} \mathrm{C}$. One of the reservoirs was filled to a level higher than the upper boundary of the channel end and left open to the atmosphere. The other reservoir was completely filled, sealed and was connected to a syringe pump (Legato200 series), which was used to impose steady flow in the channel by withdrawing oil at a constant flow rate $Q^{*}$. In order to form a finite air bubble inside the channel, the level of oil in the open reservoir was reduced so that air could be infused into the channel upon withdrawal at low flow rate of a small volume of oil from the other end of the channel. The open reservoir was then refilled through its bleed hole to avoid further air entering the system. Moreover, the size of the bubble could be decreased by positioning the edge of the bubble at the upstream end of the channel and allowing a small volume of air to escape through the bleed hole in the reservoir upon infusion of liquid at low flow rate. Air fingers were generated by keeping a low volume of oil in the open reservoir and fully opening the bleed hole so that air continuously entered the channel on removal of oil via the syringe pump.

Prior to propagation at constant flow rate $Q^{*}$, the bubble was placed in a $5 \mathrm{~cm}$ long constricted channel that spanned the width of the rail at the upstream end of the channel (Figure 1 $\mathrm{b}$ ). This channel expanded linearly over a length of $4 \mathrm{~cm}$ to reach the width of the flow channel. The bubble was propagated with a low flow rate $Q^{*}=2.0 \mathrm{ml} / \mathrm{min}$ into the diverging channel in order to allow the shape of the bubble to relax before switching off the flow for a few seconds so that the initial position of the bubble was centred and static. Note that the rail is placed along the entire length of the channel, including the transition region. All experiments were performed using this initial condition following preliminary tests showing that similar states of propagation were obtained for moderate flow rates regardless of the initial configuration. The mode of bubble propagation was found to depend on initial conditions only for the high flow rates explored in section 4.4. After each experiment, the bubble was propagated back to the inlet channel, by infusing liquid into the channel using the syringe pump. The experimental protocol was automated to enable the steady modes of bubble propagation to be characterised over a range of flow rates of $1 \leq Q^{*} \leq 70 \mathrm{ml} / \mathrm{min}$ by incrementing the flow rate in steps of $1 \mathrm{ml} / \mathrm{min}$. During these experiments, the bubble volume remained constant to within $\pm 1 \%$ [25].

Bubble propagation was monitored using a Dalsa Genie TS-M3500 camera with a 35 $\mathrm{mm} f / 1.4$ lens (Carl Zeiss $\mathrm{T}^{*}$ Distagon) mounted above the horizontal plane of the channel at a distance of $0.94 \mathrm{~m}$, which captured images of $1920 \times 218$ pixels corresponding to $271.7 \mathrm{~mm}$ $\times 30.8 \mathrm{~mm}$ (resolution of $141.5 \mu \mathrm{m} / \mathrm{px}$ ). The experiment was back-lit with a custom made LED light box made of diffusive perspex (opal 070), which produced uniform white light and was placed under the channel. Refraction of light at the bubble interface made the contour of the projected area of the bubble in top view appear dark in a light background. Sequences of $140 \leq N \leq 600$ images were captured at frames rates between $10 \geq f^{*} \geq 1$ frames 
per second, respectively, for flow rates $1 \leq Q^{*} \leq 270 \mathrm{ml} / \mathrm{min}$. Both the syringe pump and the camera were controlled in LabView; the images were processed using MATLAB R2014a routines. The edge of the bubble contour was extracted from the grey-scale images, and these binary bubble profiles were used to measure the area of the bubble in top view $A^{*}$. The size of the bubble was quantified by its effective static diameter $D^{*}$, defined as $D^{*}=2 \sqrt{A_{0}^{*} / \pi}$, which was obtained from its area $A_{0}^{*}$, measured when the bubble was in its static initial position. Hereinafter, we will refer to the static effective diameter relative to the width of the channel, $D=D^{*} / W^{*}$. The range of bubble sizes investigated in this paper is $0.35 \leq D \leq 0.87$. The smallest bubbles considered here lie towards the upper end of the range considered by FrancoGómez et al. [23], where $D$ was in the range $0.18 \leq D \leq 0.49$. Note however that the results in [23] were characterised by the ratio of bubble diameter to rail width: $d=D^{*} / w^{*}$. In terms of $d$, the present range of bubble sizes is $1.5 \leq d \leq 3.73$, while [23] explored the range $0.77 \leq d \leq 1.81$.

The position of the propagating bubble was quantified by the displacement of the centroid of its top-view contour relative to the centreline of the channel, $y_{c}^{*}$, which takes non-dimensional values $-1<y_{c}=2 y_{c}^{*} / W^{*}<1$, and by the axial position of its tip: the point on the interface with the largest $x$-coordinate. The instantaneous speed of the bubble tip, $U_{b}^{*}$, was determined by dividing the axial distance covered by the bubble tip between frames by the elapsed time, which was monitored for each frame. For most experiments in this paper, bubble deformation was mild, and states were classified as steady if both $U_{b}^{*}$ and $y_{c}^{*}$ had reached constant values by the end of the visualisation window; otherwise bubble propagation was classified as unsteady.

Steady modes of propagation will be characterised in terms of the capillary number $C a=\mu^{*} U_{b}^{*} / \sigma^{*}$. However, for unsteady modes of propagation, the bubble speed may vary in time, and so the unsteady results discussed in section 4.4 will be presented in terms of the

imposed non-dimensional flow rate defined as $Q=\mu^{*} U_{0(\exp )}^{*} / \sigma^{*}$, where $U_{0(\exp )}^{*}=Q^{*} /\left(W^{*} H^{*}\right)$ is the average speed of the fluid flow within the channel in the absence of the rail. The ratio between gravitational and surface tension forces was $B o=\rho^{*} g^{*} H^{* 2} /\left(4 \sigma^{*}\right)=0.11$, where $g^{*}$ is the acceleration due to gravity. This low value indicates that buoyancy did not affect bubble propagation significantly [23]. The importance of inertial terms relative to viscous forces is governed by the reduced Reynolds number $R e=\rho^{*} U_{0(\exp )}^{*} H^{* 2} /\left(\mu^{*} W^{*}\right)$, which has maximum value 0.2 ; thus inertia is negligible.

\section{Depth-averaged model for bubble propagation}

We model the motion of finite bubbles in a constricted channel using the same depth-averaged model as Franco-Gómez et al. [25], itself adapted from our previous models developed for air fingers [22, 23] and found to be quantitatively accurate for $\alpha=W^{*} / H^{*} \geq 40$.

The geometry of the channel cross-section used in the model is shown in Figure 2 a. 

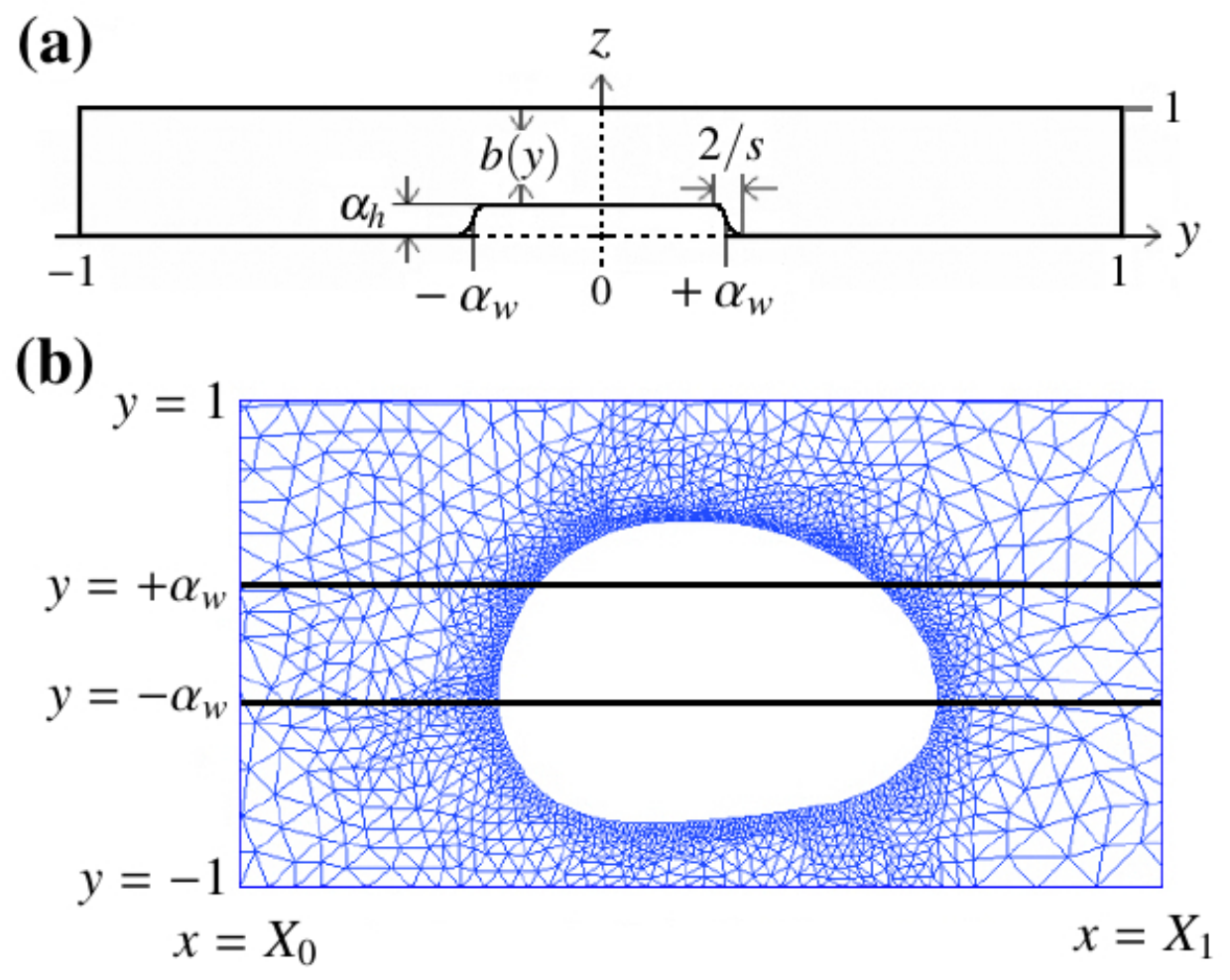

Figure 2. (a) Cross-section of the channel with a prescribed depth profile $b(y)$ where $\alpha_{w}=w^{*} / W^{*}=0.288$ is the fractional rail width, $\alpha_{h}=h^{*} / H^{*}=0.024$ the fractional rail height and $2 / s$ the width of the profile edge, with $s=40$. (b) Triangular mesh and bubble contour implemented in the numerical model. Solid black lines at $+\alpha_{w}$ and $-\alpha_{w}$ denote the edges of the rail. Figures (a) and (b) are plotted in a dimensionless coordinate system.

We introduce a Cartesian coordinate system aligned with the channel such that the in-plane coordinates $x$ and $y$ have been non-dimensionalised by $W^{*} / 2$, whereas the $z$ coordinate spanning the height of the cross-section has been non-dimensionalised by $H^{*}$. The rail is represented by a smooth tanh-profile with height given by,

$$
b(y)=1-\frac{\alpha_{h}}{2}\left[\tanh s\left(y+\alpha_{w}\right)-\tanh s\left(y-\alpha_{w}\right)\right],
$$

where $s$ is the sharpness of profile edges, and $\alpha_{h}=h^{*} / H^{*}, \alpha_{w}=w^{*} / W^{*}$ are the fractional height and fractional width of the profile, respectively.

The rail parameters used in the model are the same as those chosen based on profilometry measurements by Franco-Gómez et al. [25] and used for their study of small bubble propagation: $\alpha_{h}=0.024$ and $\alpha_{w}=0.288$. Following [23, 25], we performed the simulations with a sharpness parameter $s=40$. Here $\alpha_{h}=0.024$ is equal to the measured value of $h^{*} / H^{*}$, however $\alpha_{w}$ is larger than $w^{*} / W^{*}$. As discussed in Appendix A, if $s=40$, the choice $\alpha_{w}=0.288$ ensures that the width of the top surface of the smoothed rail matches the width 
of the top surface of the experimental rail. We provide a sensitivity analysis of the effects of rail width and sharpness in Appendix A.

The action of the syringe pump is modelled by imposing a constant pressure gradient $-G^{*} \mathbf{e}_{\mathbf{x}}$ far ahead of the bubble tip, where $G^{*}$ takes the value necessary so that the dimensional volume flux is fixed at $Q^{*}$. We non-dimensionalise the two components of the depthaveraged horizontal velocity $\mathbf{u}^{*}$ on the scale $U_{0}^{*}=Q^{*} /\left(H^{*} W^{*}\right)$, horizontal coordinates on the scale $W^{*} / 2$, time on the scale $W^{*} /\left(2 Q^{*}\right)$ and pressure on the scale $6 \mu Q^{*} / H^{* 3}$, where $\mu^{*}$ is the dynamic viscosity of the fluid. After applying the lubrication approximation [28], the governing equation for the viscous, incompressible fluid in the frame of reference moving with speed $\mathbf{U}_{b}=\left(U_{b}, 0\right)$, where $U_{b}=U_{b}^{*} / U_{0}^{*}$, is

$$
\nabla \cdot\left(b(y)^{3} \nabla p\right)=0 \quad \text { in } \Omega,
$$

where $\Omega$ denotes the fluid domain. The fluid domain is given by $X_{0} \leq x \leq X_{1},-1 \leq y \leq 1$, excluding the region occupied by the bubble, where $X_{0}$ and $X_{1}$ are truncation coordinates behind and ahead of the centre of the bubble (Figure $2 \mathrm{p}$ ).

The conditions at the bubble interface $\mathbf{R}=(x, y)$ and on the channel boundaries are

$$
\begin{aligned}
& \hat{\mathbf{n}} \cdot \frac{\partial \mathbf{R}}{\partial t}+\hat{\mathbf{n}} \cdot \mathbf{U}_{b}+b^{2} \hat{\mathbf{n}} \cdot \nabla p=0 \quad \text { on } \quad \partial \Omega_{b}, \\
& p_{b}-p=\frac{U_{b}}{3 \alpha C a}\left(\frac{1}{b(y)}+\frac{\kappa}{\alpha}\right) \quad \text { on } \quad \partial \Omega_{b}, \\
& \frac{\partial p}{\partial y}=0 \quad \text { on } \quad y= \pm 1, \\
& \frac{\partial p}{\partial x}=-G \quad \text { on } \quad x=X_{0}, \quad \frac{G}{2} \int_{-1}^{1} b^{3}(y) \mathrm{d} y=1 . \\
& p=0 \quad \text { on } \quad x=X_{1},
\end{aligned}
$$

where $\partial \Omega_{b}$ denotes the bubble boundary with unit normal $\hat{\mathbf{n}}$ directed into the fluid. The interface conditions in the moving frame have been previously derived [23] and the only difference from the interface conditions in the fixed lab frame [22], is in equation (3), which adds the velocity of the frame, $\mathbf{u}=-\mathbf{U}_{b}-b^{2} \boldsymbol{\nabla} p$, into the kinematic boundary condition $\hat{\mathbf{n}} \cdot \partial \mathbf{R} / \partial t=\hat{\mathbf{n}} \cdot \mathbf{u}$. Note that (5) implies that there is no normal velocity at the channel side walls.

The $\partial \mathbf{R} / \partial t$ term in (3) is the only time derivative in the problem and prescribes the unsteady evolution of the bubble. In this work, we primarily present steady solutions, computed by setting $\partial \mathbf{R} / \partial t=0$. However, the time-derivative term in the equations is important for the linear stability calculations presented throughout.

The dynamic boundary condition (4) is the non-dimensional form of the Young-Laplace equation, where $p_{b}$ is the pressure inside the bubble and $\kappa$ is the non-dimensional curvature 
of the interface in the $(x, y)$ plane, which we shall term the in-plane curvature. The other component of curvature, $1 / b(y)$, will be termed the transverse curvature. Note that in the case $b(y)=1$, the geometry of the channel reduces to a rectangular Hele-Shaw channel, and the equations reduce to those for classic Hele-Shaw flow. The bubble velocity $U_{b}$ is an unknown, chosen so that that the geometric centroid of the bubble is fixed at $x=0$; this velocity may vary in time. The fluid pressure is fixed to be zero far ahead of the bubble, which means that the bubble pressure $p_{b}$ is also an unknown with the associated constraint that the bubble volume must remain a prescribed constant, $V_{0}$, during the evolution

$$
\iint_{\Omega_{b}} b(y) \mathrm{d} A=V_{0}=\frac{\pi D^{2}}{4},
$$

assuming that the bubble occupies the full height of the channel. In the model, we neglect the effects of the thin films that separate the bubble from the top and bottom boundaries of the channel in this volume constraint and also in the two boundary conditions at the bubble interface.

The model is solved using the finite element library oomph-lib [29] and implementation details are given in [22, 23, 25]. Where needed, linear stability calculations are performed by formulating and solving a generalised eigenvalue problem within the oomph-lib framework, as discussed in some detail in [22]. The centroid position $y_{c}$ is calculated directly from the bubble boundary, using the same algorithm as for the experimental observations.

Naturally, the model does not capture all the features of the three-dimensional system. As mentioned above, our model neglects any thin films above and below the bubbles. A number of formulations have been proposed to correct for the presence of the film, but are generally only appropriate if the film thickness is spatially uniform. These corrections take the form of an increased propagation speed [15] due to a modified mass balance at the propagating interface, or a reduction of the effective lateral curvature in the normal pressure jump by a factor of $\pi / 4$ [30, 31] . We note that this latter result only applies at small $C a$, large $\alpha$ and for a rectangular channel. It is difficult to apply these corrections in our model as we do not operate fully in this asymptotic regime, the presence of the rail means it is unlikely that film thicknesses are spatially uniform, and we do not have a predictive model for these thicknesses. Thus, any such correction would be necessarily ad-hoc. We note that for the case of finger propagation with a similar depth-profile to that explored here, we have previously shown that the uncorrected model is in quantitative agreement with the experimental results when $C a \lesssim 0.012, \alpha_{h} \leq 0.12$ and $\alpha \gtrsim 40$.

The low order of the model means that we can satisfy only one boundary condition on each part of the fluid boundary, and we choose to apply the normal pressure jump at the bubble and the no-penetration condition at the side wall. This means that we cannot apply the no-slip boundary condition along the channel side walls, nor the condition of zero tangential stress at the bubble interface. It would be possible to apply these boundary condition by including higher order derivatives in the model, such as via the Brinkman equations, but these 
Brinkman effects are just one of a number of possible corrections (including the thin films suggested above), and we prefer to focus on the uncorrected model presented here. Again, we note that quantitative agreement has been reached for air fingers in wide aspect ratio systems without incorporating the tangential stress boundary condition.

We would expect the no-slip and no-tangential stress conditions to be most significant when the bubble boundary is very close to the channel walls (e.g. the inset image marked (a) in Figure $3 \mathrm{~b}$ ). In fact, we found that we were unable to compute asymmetric steady solutions to the model at very low $C a$; instead each solution branch terminates at a finite value of $\mathrm{Ca}$, marked in the figures by a filled circle. This minimum value of $C a$ does not appear to correspond to a bifurcation point, and the behaviour of the bubbles in this regime is similar to that observed experimentally.

\section{Results}

\subsection{From finger to bubble propagation}

We begin by presenting new results for the propagation of a finger (a semi-infinite bubble), in the presence of the rail of modest height used in this study $\left(\alpha_{h}=0.024\right.$, i.e. $2.4 \%$ of the height of the channel). Steady modes of finger propagation are quantified in Figure $3 \mathrm{a}$ in terms of the offset $\delta$ of the finger tip from the axial centreline of the channel (see figure caption for definition of $\delta$ ) as a function of the capillary number [23]. The finger is symmetric about the centreline of the channel in the capillary-static limit, and this symmetric finger propagates stably as $\mathrm{Ca}$ is increased up to a critical value of the capillary number, $C a_{\mathrm{c} 2(\exp )} \approx 6.1 \times 10^{-3}$, at which point the finger undergoes a supercritical symmetry-breaking bifurcation to an asymmetric finger. The unavoidable small inherent bias present in the experimental channel promotes asymmetric propagation primarily on one side of the rail. The open symbols denote fingers that were still transiently evolving towards an off-centre steady state when they reached the end of the experimental channel. These points, which are typically clustered just past the bifurcation point, reflect the critical slowing down of finger evolution near the bifurcation point. Transient states were not observed for $C a<C a_{\mathrm{c} 2(\exp )}$ because both the initial position of the finger and the stable steady state were centred.

Steady numerical solutions of the depth-averaged model are shown in Figure 3 a by a solid line. The results are qualitatively similar to those previously presented by [23], but in that work $\alpha_{w}$ was $20 \%$ smaller. Comparison of the results for the two rail widths in Appendix $\mathrm{A}$ indicates that the narrower rail is associated with a slightly larger value of the critical

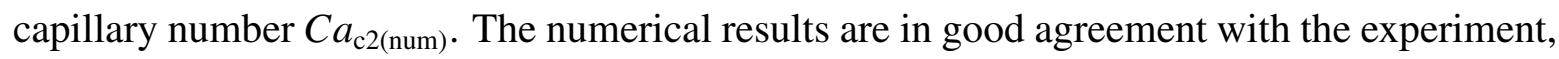
although the bifurcation point occurs for $C a_{\mathrm{c} 2(\mathrm{num})} \simeq 6.8 \times 10^{-3}$, which is larger than $C a_{\mathrm{c} 2(\exp )}$ by approximately $11 \%$. This result, obtained for $\alpha=30$, is consistent with the findings of Franco-Gomez et al. [23], who demonstrated quantitative agreement between experiment and 

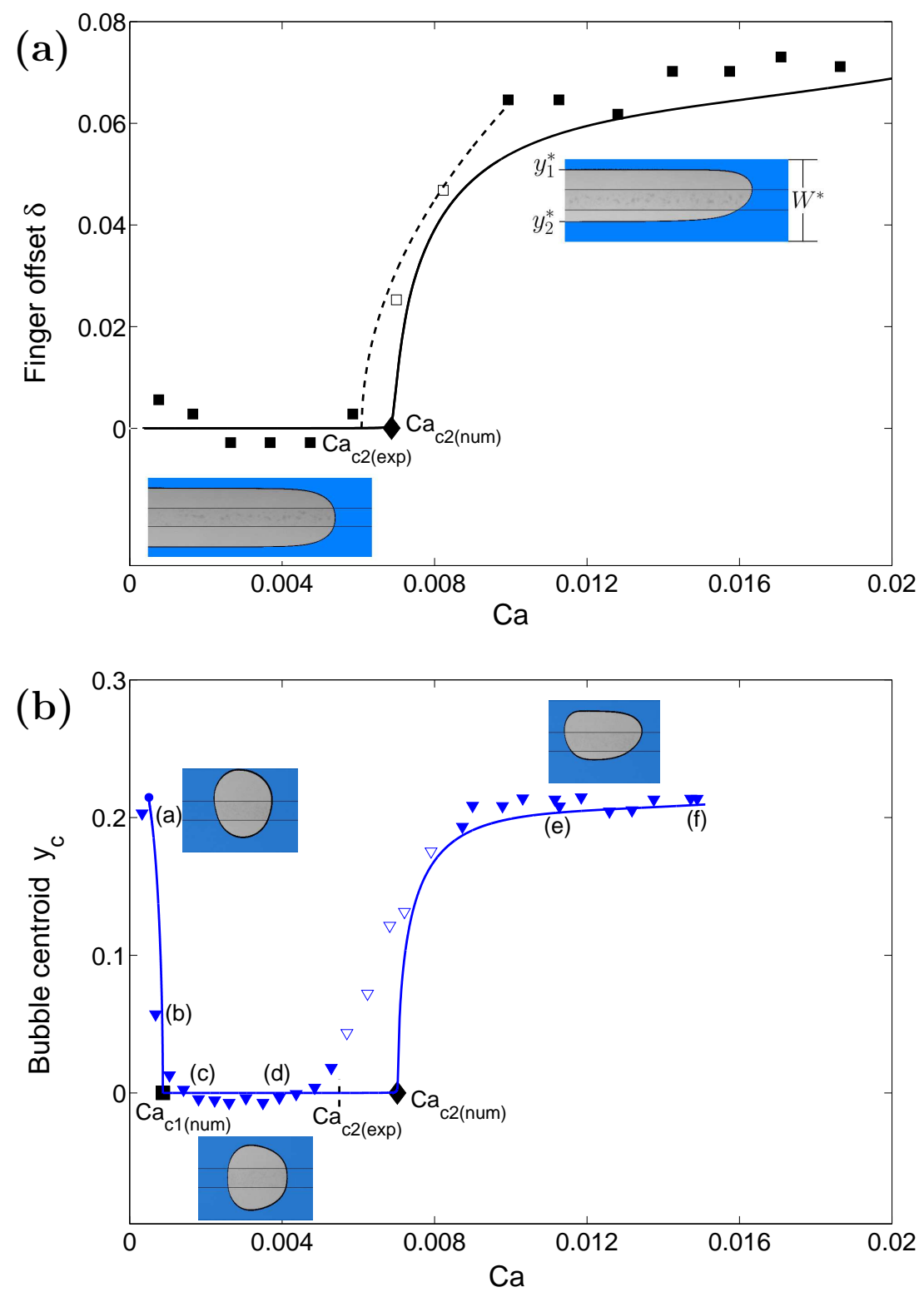

Figure 3. Comparison between experimental (symbols) and numerical (solid lines) steady modes of propagation in a channel with aspect ratio $\alpha=30, \alpha_{h}=0.024$, as a function of capillary number $C a$. The measured rail width is $\alpha_{w}=0.233$; for the numerical model, we use $\alpha_{w}=0.288$ and sharpness parameter $s=40$. Filled symbols correspond to steady modes of propagation and open symbols denote modes of propagation that do not reach a steady state within the length of the channel. (a) Finger propagation, where the finger offset is defined as $\delta=\left(y_{1}^{*}+y_{2}^{*}\right) /\left(2 W^{*}\right)$; for large bubbles $2 \delta \approx y_{c}$ where $y_{c}$ is the centroid offset. The symmetric finger loses stability to an asymmetric finger through a supercritical pitchfork bifurcation. The experimental critical capillary number, $C a_{\mathrm{c} 2(\mathrm{exp})}$, was determined by fitting a square-root function to data points just past the bifurcation point (dashed line) [19]. (b) Propagation of a bubble of effective static diameter $D=0.76\left(D^{*}=22.8 \mathrm{~mm}\right)$. The black symbols denote the locations of supercritical pitchfork bifurcations. The filled circle on the computed stable propagation modes indicates the smallest value of $\mathrm{Ca}$ for which a solution could be computed. 
model, with less than 5\% discrepancy, for aspect ratios $\alpha \geq 40$. However, for $\alpha=20, C a_{\mathrm{c} 2 \text { (num) }}$ exceeded $C a_{\mathrm{c} 2(\exp )}$ by approximately $50 \%$. This dependence on $\alpha$ is consistent with the model assumptions; the validity of the two-dimensional model relies on sufficiently high aspect ratio channels, in addition to small occlusion heights and small values of $C a$.

The loss of symmetry of the propagating mode is due to the variation of local viscous resistance across the channel due to the presence of the rail. For a near-uniform axial pressure gradient, both fluid and interface will propagate faster in the off-rail regions, but this speed differential is resisted by surface tension acting through the in-plane curvature of the finger to stabilise the finger in a centred, symmetric configuration over the rail. For sufficiently large flow rates, the viscous forces dominate and variations in the local interface speed near the finger tip are able to drive the finger into the off-rail regions, resulting in the observed symmetry-breaking of the finger at a critical value of $C a$ [22, 23].

In Figure 3b, we present the steady propagation modes of a finite, but relatively large bubble, started from a centred initial position with $D=0.76$, where the centroid of the bubble is plotted as a function of $\mathrm{Ca}$. Analogously to the finger case shown in Figure $3 \mathrm{a}$, the bubble loses symmetry through a supercritical pitchfork bifurcation $(\boldsymbol{\nabla})$, at similar values of $C a_{\mathrm{c} 2(\exp )}$ and $C a_{\mathrm{c} 2 \text { (num) }}$ to the finger. The off-centre propagation of both bubbles and fingers at high flow rates is supported by the reduced viscous resistance in the deeper parts of the channel, which dominates over capillary effects when $C a$ is large. However, a key difference in the bubble scenario is the absence of a symmetric capillary-static state. The bubble can only adopt a stable symmetric capillary-static configuration once its volume is sufficient that it spans the entire width of the channel [21]. When the volume of the bubble is reduced below this threshold, the symmetric capillary-static state is no longer stable to lateral perturbations because the reduction in transverse curvature when the bubble expands into one of the deeper side channels on either side of the rail drives a flow that pushes the bubble further to the side until an asymmetric equilibrium is reached; see Section 4.2 for further discussion. The introduction of axial flow, however, changes the behaviour: for small values of $C a$, for which variations in viscous resistance across the channel are small compared to surface tension, the bubble inclines so that its tip is nearer the centreline, which acts to drive the bubble towards an on-rail position [25]. Thus, the capillary-static induced flow and viscous-pressure-driven flows act in opposite directions and for moderately large flow rates, the centred mode of propagation is stabilised, as shown in Figure 3 b. The flow-induced stabilisation of this symmetric mode takes place through a supercritical pitchfork bifurcation (ם) at $C a_{\mathrm{c} 1 \text { (num) }}<C a_{\mathrm{c} 2 \text { (num) }}$, which is in good agreement with the experimental value. Hence, the bubble propagates symmetrically about the centreline of the channel over a limited range of $\mathrm{Ca}$ between the two bifurcations. Note that near the bifurcation, the experimental data for bubbles is less well approximated by a square-root function than the results for fingers, suggesting that the bubbles are more sensitive to imperfections.

Figure 4 shows a direct comparison of experimental and numerical bubble shapes 
(a)

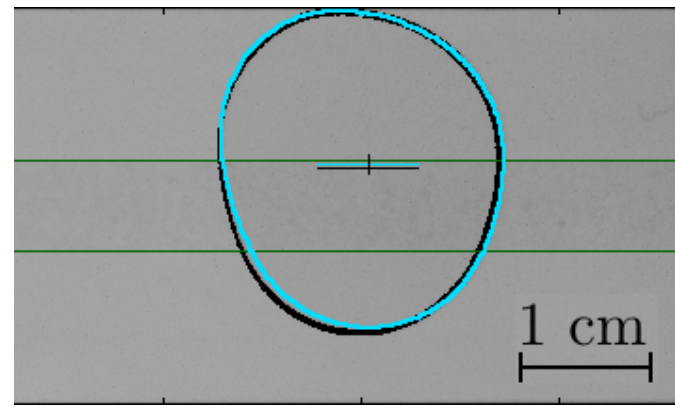

(b)

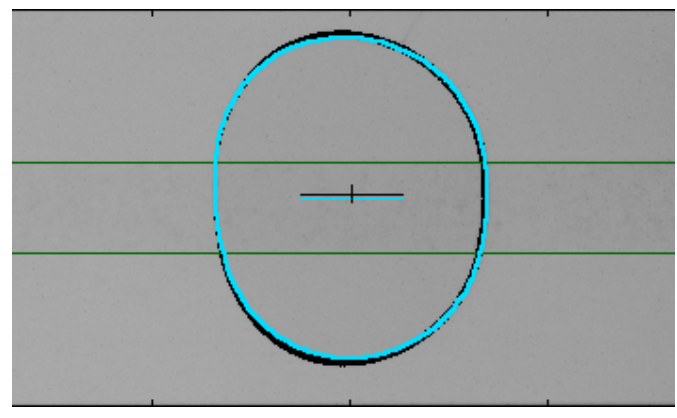

(c)

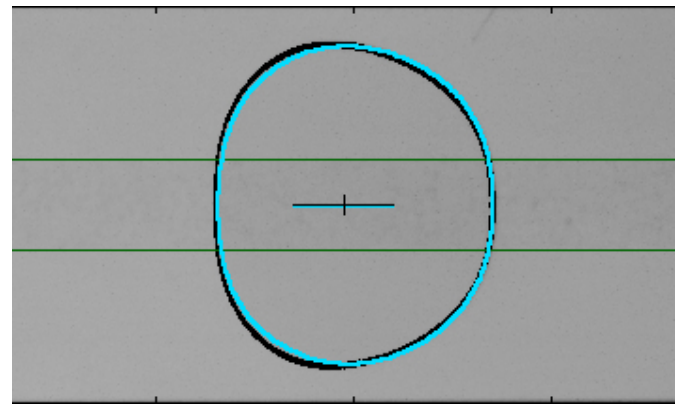

(d)

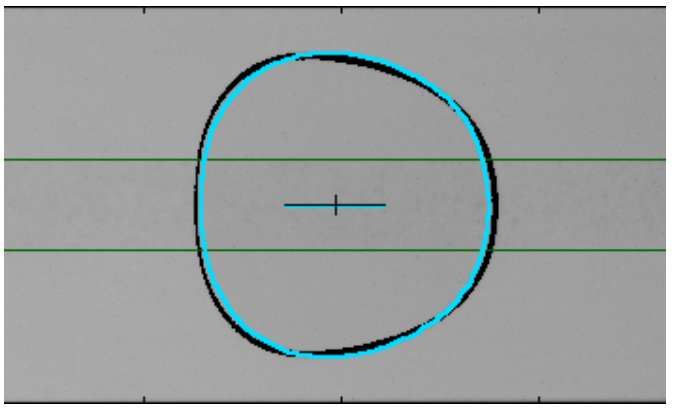

(e)

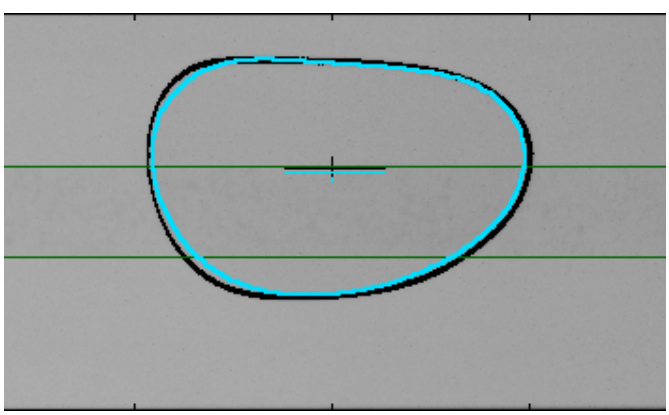

(f)

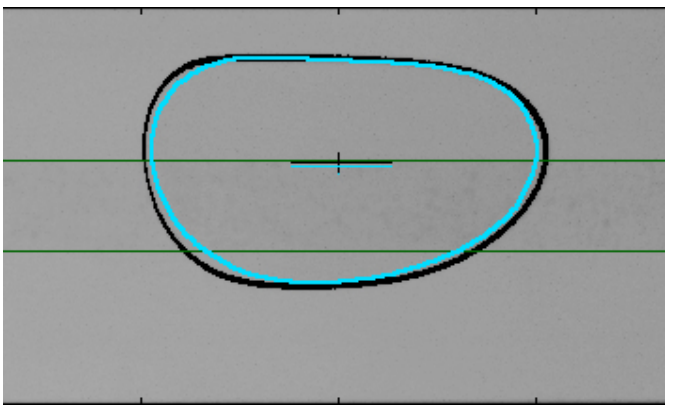

Figure 4. Direct comparison between experimental and numerical bubble outlines for increasing values of $C a$, bubbles with static effective diameter $D=0.76\left(D^{*}=22.8\right.$ $\mathrm{mm})$. The computed bubbles shapes are shown with solid cyan lines, experimental bubble outlines with solid black lines, and the edge of the rail is highlighted with a solid green line. The capillary numbers (dimensional flow rates) are: (a) $C a_{\text {num }}=$ $5.34 \times 10^{-4}, C a_{\text {exp }}=3.26 \times 10^{-4}\left(Q^{*}=1 \mathrm{ml} / \mathrm{min}\right) ;\left(\right.$ b) $C a_{\text {num }}=7.74 \times 10^{-4}$, $C a_{\text {exp }}=6.80 \times 10^{-4}\left(Q^{*}=2 \mathrm{ml} / \mathrm{min}\right) ;$ (c) $C a=1.81 \times 10^{-3}\left(Q^{*}=5 \mathrm{ml} / \mathrm{min}\right)$; (d) $C a=3.92 \times 10^{-3}\left(Q^{*}=10 \mathrm{ml} / \mathrm{min}\right)$; (e) $C a=1.12 \times 10^{-2}\left(Q^{*}=23 \mathrm{ml} / \mathrm{min}\right)$; and (f) $C a=1.49 \times 10^{-2}\left(Q^{*}=30 \mathrm{ml} / \mathrm{min}\right)$. Note that in (a) and (b) only, the numerical value of $C a$ is chosen so that the shapes have similar offset values; otherwise the same value of $C a$ is used for both experimental and numerical results. 
corresponding to the data shown in Figure $3 \mathrm{~b}$, with the numerical bubbles shown by cyan outlines overlaid on experimental images where the bubble boundary is visible as a black line. The scale for the images is chosen to match the channel width, with the downstream location chosen to match the axial position of the centroid. Both the lateral components of the centroids and the overall shapes of the experimental and numerical bubbles outlines are in good agreement for the three observed propagation modes: asymmetric low- $\mathrm{Ca}$, Figure $4 \mathrm{a}, \mathrm{b}$; symmetric, Figures 4r,d; and asymmetric high- $\mathrm{Ca}$, Figures 4 e,f.

Although the bubble shapes are in good agreement, the projected area of the experimental bubble is consistently larger than that of the numerical bubble, and this discrepancy increases with increasing $\mathrm{Ca}$. As $\mathrm{Ca}$ increases in the experiment, the thickness of the liquid films separating the bubble from the top and bottom boundaries of the channel increases [25, 31], which in turn enlarges the projected area of the experimental bubble. The difference in bubble projected areas between experiment and model arises because the model does not account for the presence of these liquid films, instead assuming that the bubble always occupies the whole height of the channel for the purpose of applying the volume constraint. The experimental projected area exceeds the numerical one by only $2.6 \%$ in Figure $4 \mathrm{a}$, but this value rises to $10.4 \%$ in Figure $4 \mathrm{f}$. We refer to Appendix B for systematic measurements of bubble area variations with $C a$ over the entire range of $C a$ investigated.

\subsection{Effect of bubble size on the steady modes of bubble propagation}

Having established the bifurcation sequence for varying $C a$ that underpins the propagation of finite bubbles from a centred initial position, for a single moderately sized bubble, we show the evolution of this picture for decreasing bubble size in Figure 5 . For very small values of $\mathrm{Ca}$, the displacement of the bubble centroid from the centreline increases significantly as the bubble size is reduced, suggesting increasingly asymmetric capillary-static configurations. This is simply because a greater proportion of a smaller bubble fits within the off-rail region. In addition, smaller bubbles have larger in-plane curvatures, and thus the surface-tensioninduced pressure variations are greater than for larger bubbles. Hence, larger viscous forces are in turn required in order to stabilise symmetric modes of propagation. This means that the critical capillary number associated with the first supercritical pitchfork bifurcation, which stabilises the steady symmetric bubble, increases as the bubble size is reduced. In fact, the computed values of $C a_{\mathrm{c} 1 \text { (num) }}$ for the cases shown in Figure 5 closely match the experimental values of $C a_{\mathrm{c} 1(\exp )}$. Moreover the centroid positions of the asymmetric bubbles at very low values of $C a$ below $C a_{\mathrm{c} 1}$ remain well captured by the numerical computations. The experimental data at small $C a$ for the three largest bubbles indicate that $C a_{\mathrm{c} 1}$ decreases as the bubble size increases, suggesting that a capillary-static symmetric bubble would eventually be recovered if the bubble size was further increased, as expected based on the behaviour of semi-infinite air fingers. 


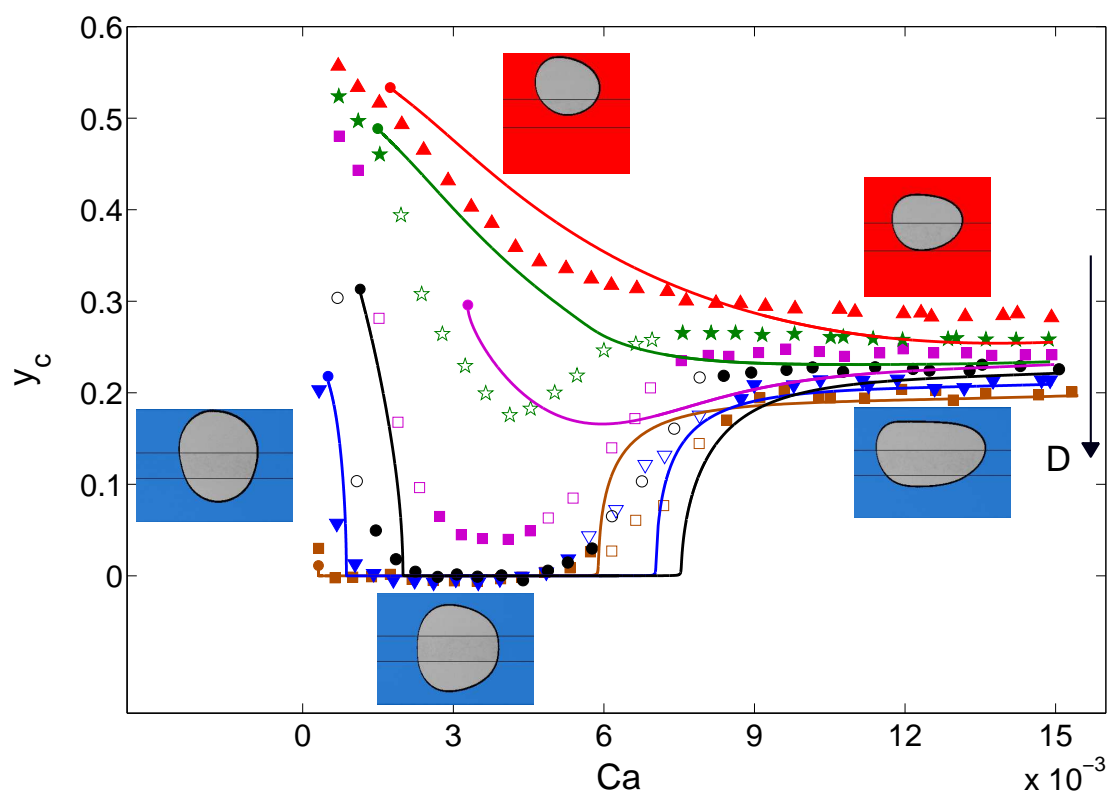

Figure 5. Comparison between experimental and numerical bubble centroid displacements from the centreline, $y_{c}$, as a function of $C a$, for different bubble sizes quantified by their static non-dimensional (dimensional) diameters: $(\boldsymbol{\Delta}) D=0.50$ $\left(D^{*}=14.9 \mathrm{~mm}\right) ;(\star) D=0.55\left(D^{*}=16.5 \mathrm{~mm}\right) ;(\square) D=0.59\left(D^{*}=17.8\right.$ $\mathrm{mm}) ;(\bullet) D=0.67\left(D^{*}=20.1 \mathrm{~mm}\right) ;(\nabla) D=0.76\left(D^{*}=22.8 \mathrm{~mm}\right)$ and $(\square)$ $D=0.87\left(D^{*}=26.1 \mathrm{~mm}\right)$. Each data point corresponds to a bubble propagating under constant imposed flow rate from an initially centred position. The numerically determined steady states corresponding to these bubble sizes are shown with solid lines of matching colour. Note that for small values of $\mathrm{Ca}$ the curves are shifted monotonically towards smaller values of $y_{c}$ with increasing bubble diameter. The filled circle marking the lower $\mathrm{Ca}$ extremity of each line correspond to the smallest value of $C a$ for which a solution could be computed. The channel parameters are $\alpha=30, \alpha_{h}=0.024, \alpha_{w}=0.288$ and $s=40$.

The three smaller bubbles in Figure 5 do not exhibit symmetric propagation at any $\mathrm{Ca}$. This is because the viscous forces required to compensate for the destabilising effect of surface tension forces become sufficiently large that they induce significant variations in flow across the channel. In response to a perturbation of the bubble, the flow variations have the effect of tilting the bubble tip away from the rail and into the deeper side channel, so that stabilisation of the symmetric mode of propagation is prevented [25]. The disappearance of the stable symmetric bubble occurs through the coalescence of the two nearby pitchfork bifurcations for a critical bubble diameter $D_{c}$, in the region $0.59<D_{c}<0.67$ (i.e. between the magenta and black symbols in Figure 5). For bubbles below this critical diameter, the bubble always propagates in an off-centred position, but there is still a noticeable shift at intermediate $\mathrm{Ca}$ towards the centre of the channel due to the stabilising effect of viscous 
(a)

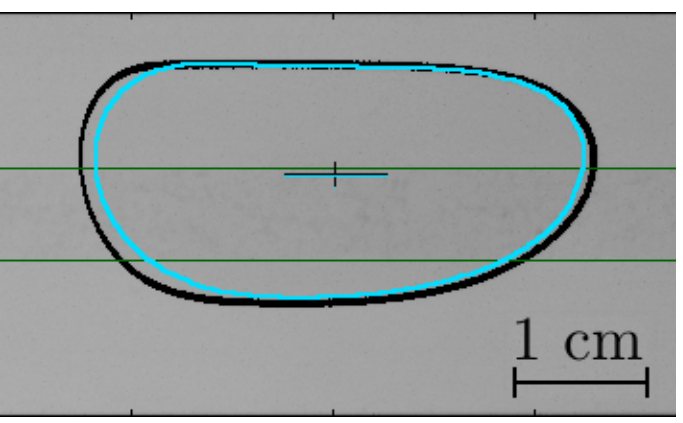

(b)

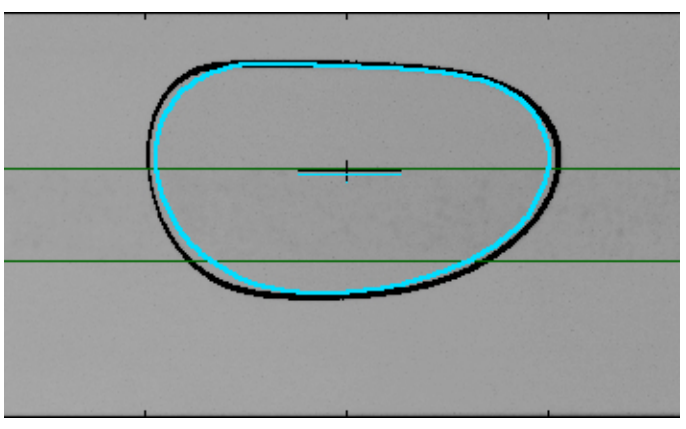

(c)

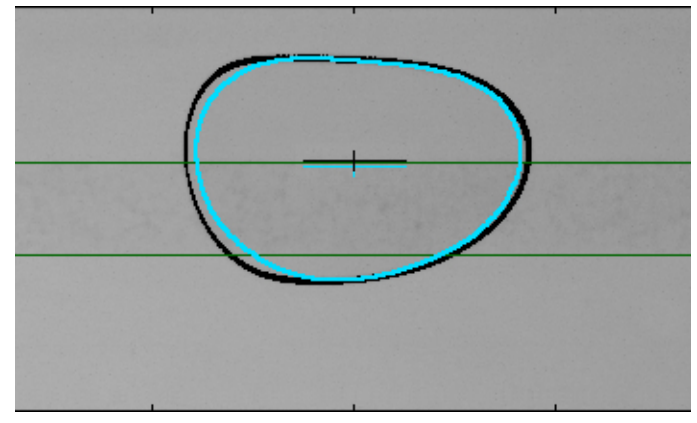

(d)

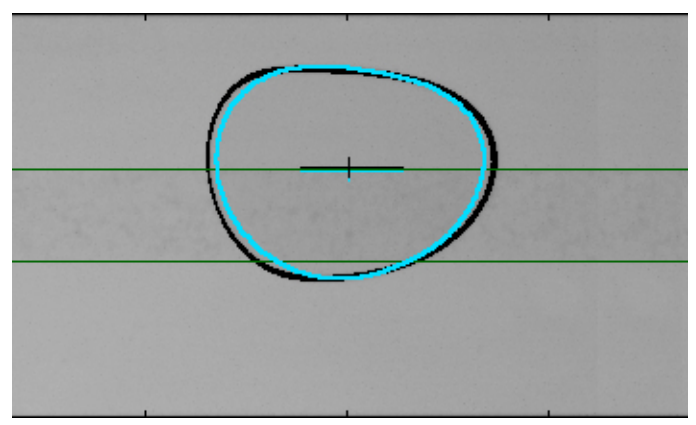

(e)

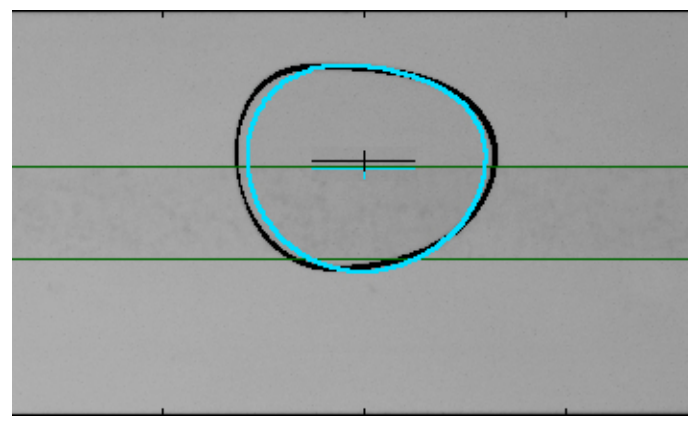

(f)

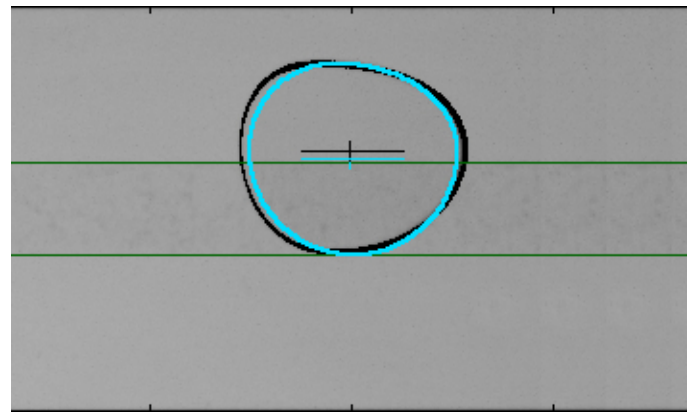

Figure 6. Direct comparison between experimental and numerical bubble outlines for decreasing bubble sizes. The bubbles were propagated with the highest values of $C a \simeq 1.5 \times 10^{-2}\left(Q^{*}=30 \mathrm{ml} / \mathrm{min}\right)$ shown in Figure 5. The computed bubbles shapes are shown with solid cyan lines, experimental bubble outlines are plotted with solid black lines, and the edge of the rail is highlighted with a solid green line. The bubble sizes are quantified by the effective nondimensional (dimensional) diameters of the static bubbles: (a) $D=0.87\left(D^{*}=26.1 \mathrm{~mm}\right)$; (b) $D=0.76\left(D^{*}=22.8 \mathrm{~mm}\right)$; (c) $D=0.67\left(D^{*}=20.1 \mathrm{~mm}\right) ;(\mathrm{d}) D=0.59\left(D^{*}=17.8 \mathrm{~mm}\right) ;(\mathrm{e}) D=0.55\left(D^{*}=16.5\right.$ $\mathrm{mm})$ and (f) $D=0.50\left(D^{*}=14.9 \mathrm{~mm}\right)$. 
forces. When $C a$ becomes sufficiently large, $y_{c}$ increases again and eventually reaches an approximately constant value for sufficiently large $\mathrm{Ca}$. The centroid positions $y_{c}$ of the experimental asymmetric states on the right-hand side of Figure 5 are accurately captured by the model for the three largest bubbles. However, the smaller three bubbles exhibit discrepancies in $y_{c}$ at these large $C a$, most likely due to enhanced three-dimensional flow features for reduced bubble aspect ratios.

The coalescence of the two pitchforks leads to a stable asymmetric branch for both the experimental and numerical results shown in Figure 5. However, these two branches are not in quantitative agreement. This is in part due to the sensitivity of the system to small bubble size changes in this regime, but also because of the long duration of transient evolution of the propagation modes, which means that steady states are not typically reached within the length of the experimental channel. These long transients, together with unavoidable imperfections in the experimental system, mean that the bifurcation regions for the three larger bubbles differ between the model and experiments, particularly near $C a_{\mathrm{c} 2}$. In the model calculations the location of $C a_{\mathrm{c} 2(\mathrm{num})}$ varies non-monotonically with bubble size, which is due to the subtle and non-trivial nature of the interplay between viscous and surface tension forces that leads to destabilisation of the symmetric solution. It is not possible to determine any similar trends for the experimental data which all agree, within experimental error, for the three largest bubbles in the region near $C a_{\mathrm{c} 2(\exp ) \text {. }}$

A direct comparison between experimental and numerical bubble outlines for the bubbles sizes shown in Figure 5 is presented in Figure 6 at the largest value of $C a$ considered, $C a \simeq 1.5 \times 10^{-2}$. As was the case in Figure 4, the presence of thin liquid films above and below the bubble lead to the experimental projected area exceeding that of the numerical bubble. The two areas differ by an approximately constant factor of $10.6 \pm 0.9 \%$. In Appendix $\mathrm{B}$. we track bubble area across the range of $C a$ and $D$ used in Figure 5, and find that the area ratio increases with $C a$, but is largely independent of bubble size. This implies that the average film thickness is independent of bubble size at fixed capillary number. Despite this discrepancy in area, however, the numerical bubble shapes in Figure 6 closely match those for the experimental bubbles, and the crosses denoting the centroid positions of the numerical and experimental bubbles highlight the small differences in $y_{c}$. As previously discussed in relation to Figure 5, at large $C a$ the discrepancies in $y_{c}$ increase only modestly as the bubble size is decreased.

\subsection{Numerical bifurcation diagram}

In addition to predicting the stable modes of steady propagation of the bubble, the numerical simulations can also capture unstable solutions. Steadily propagating bubbles are quantified in Figure 7 in terms of their centroid position (a) and non-dimensional propagation speeds as functions of $\mathrm{Ca}(\mathrm{b}, \mathrm{c})$. While the two asymmetric branches emerging from each pitchfork 


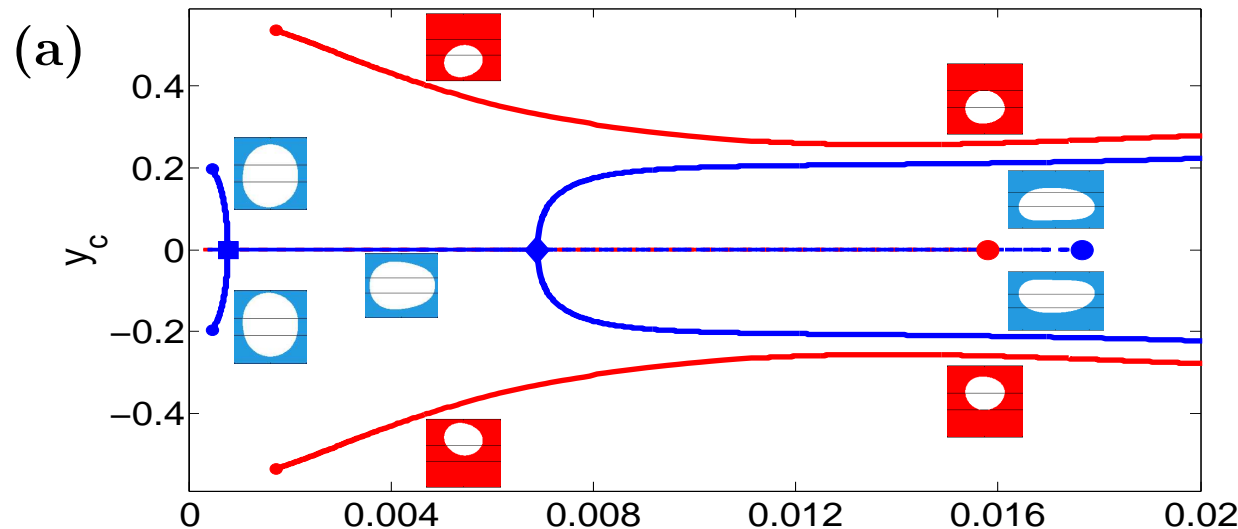

(b)
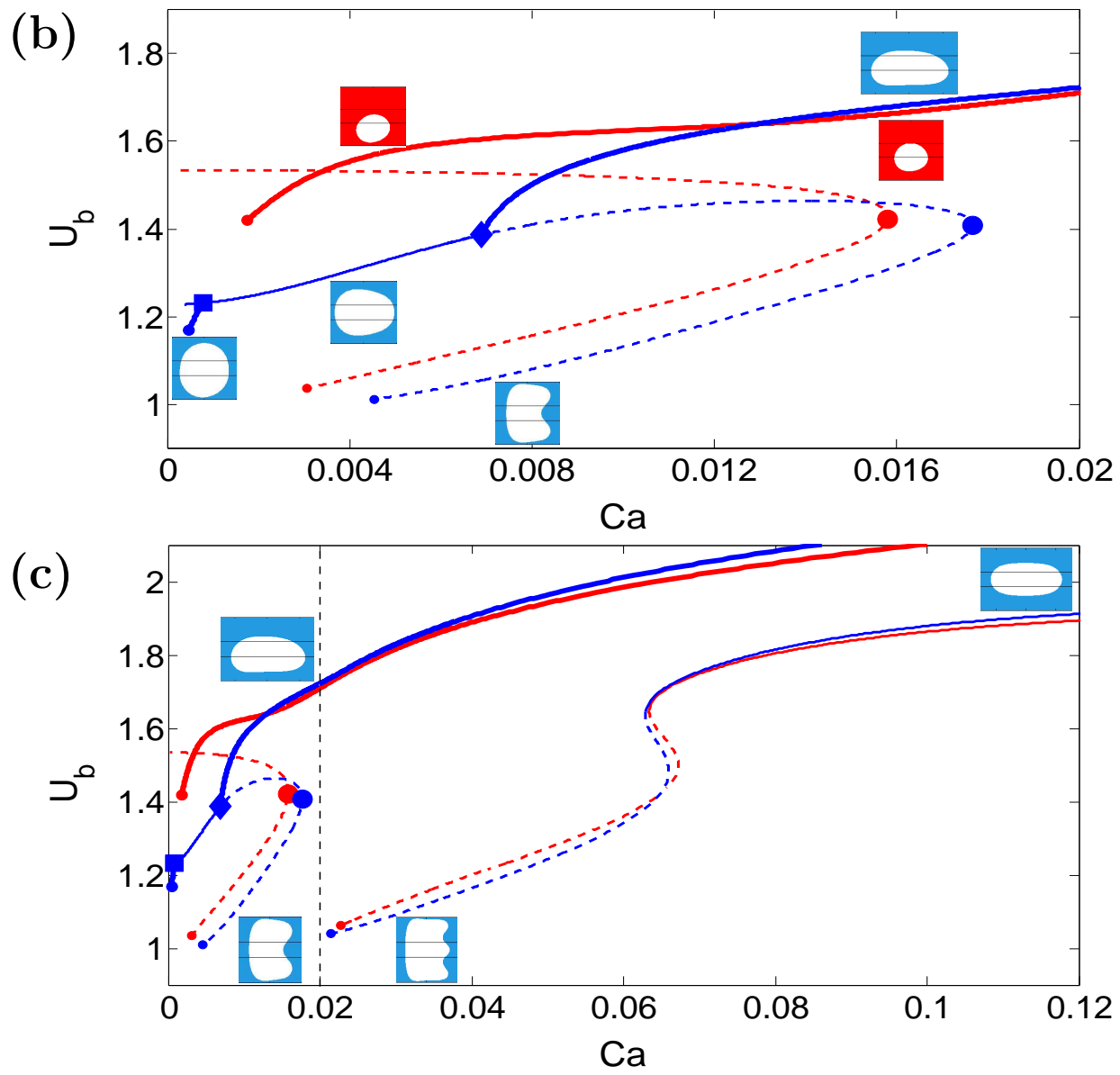

Figure 7. Numerical simulations of (a) centroid position $y_{c}$ and $(\mathrm{b}, \mathrm{c})$ nondimensional bubble speed $U_{b}$ as a function of capillary number $C a$ for bubble sizes $D=0.5$ $\left(D^{*}=15 \mathrm{~mm}\right)$ in red and $D=0.77\left(D^{*}=23.2 \mathrm{~mm}\right)$ in blue. Solid thick lines correspond to asymmetric stable states, solid thin lines to symmetric stable states and dashed thin lines to symmetric unstable states The channel parameters are $\alpha=30$, $\alpha_{h}=0.024, \alpha_{w}=0.288$ and $s=40$. Projection (a) shows the pitchfork bifurcation to off-centre branches, but there are also multiple centred branches, which are revealed in projection (b). At larger $C a$ in (c), there is a disconnected symmetric branch including a family of stable propagation modes and hence both symmetric and asymmetric propagation modes are stable for $C a \gtrsim 0.06$. 
bifurcation are differentiated in projection (a), they collapse onto a single line in projection (b). Stable (unstable) modes of propagation are shown with solid (dashed) lines, respectively. The bifurcation diagram shown in Figure $7 \mathrm{a}$ spans a similar range of capillary numbers to that studied in section 4.2. The blue line corresponds to the same large bubble $(D=0.77)$ as in Figures $3 \mathrm{~b}$ and 4 and is also shown in blue in Figure 5. At very low values of $C a$, this large bubble propagates stably in an asymmetric configuration. The asymmetric solution branch disappears at the first supercritical pitchfork bifurcation point ( $\square$ ), enabling stable symmetric bubbles to propagate as $\mathrm{Ca}$ is increased further until the second supercritical pitchfork bifurcation point $(\diamond)$, where the symmetric mode loses stability in favour of an asymmetric mode, as discussed in section 4.1. In contrast, the smaller bubble (red lines, $D=0.5$ ) propagates stably in an asymmetric configuration over the entire range of $\mathrm{Ca}$ investigated numerically. In this case, the symmetric branch is disconnected from the asymmetric branch and remains unstable for all values of $C a$.

For both bubbles, the unstable symmetric branch undergoes a saddle-node bifurcation at a limit point to an unstable dimpled bubble solution, the first Romero-Vanden-Broeck (RVB) solution, which was originally uncovered in the context of Saffman-Taylor fingering [32-34]. In Figure 7k, we increase the range of capillary numbers by a factor of six, and capture the second RVB solution, which exhibits two dimples at its tip but is also unstable. Green et al. [18] have recently investigated bubble shapes in unbounded Hele-Shaw flow and have found a countably infinite number of disconnected solution branches, each corresponding to a particular number of dimples at the bubble tip. At fixed dimensionless speed $U_{b}=U_{b}^{*} / U_{0}^{*}$, the branches occur at increasing $\mathrm{Ca}$ for increasing dimple number. We expect similar solution branches to exist in our system, despite the presence of the bounding channel walls and the rail, but we have not attempted to compute them. With increasing $C a$, the second RVB solution in Figure $7 \mathrm{f}$ undergoes two saddle-node bifurcations, while its shape evolves towards a round tipped bubble. These bifurcations mean that a symmetric mode of propagation is restabilised at large values of $C a$, analogously to the previously studied finger propagation [23]. In experiments, the observed steady propagation modes should be stable, but we show in section 4.4 that unstable solutions do arise transiently.

\subsection{Unsteady bubble propagation at large flow rates}

Thus far, we have focused on a range of bubble sizes and capillary numbers for which an initially centred bubble rapidly evolves towards a stable steady mode of propagation, which may or may not be symmetric about the centreline of the channel. The second pitchfork bifurcation occurs at $C a_{\mathrm{c} 2(\exp )} \simeq 6 \times 10^{-3}$ as shown in Figure 5 , and beyond this point the only steady modes of propagation we have observed experimentally are asymmetric. We also found numerically that a steady symmetric mode of propagation is stabilised above the much larger value $C a \approx 9 \times 10^{-2}$ (Figure 7). Hence, at these values of $C a$ the model predicts that the 


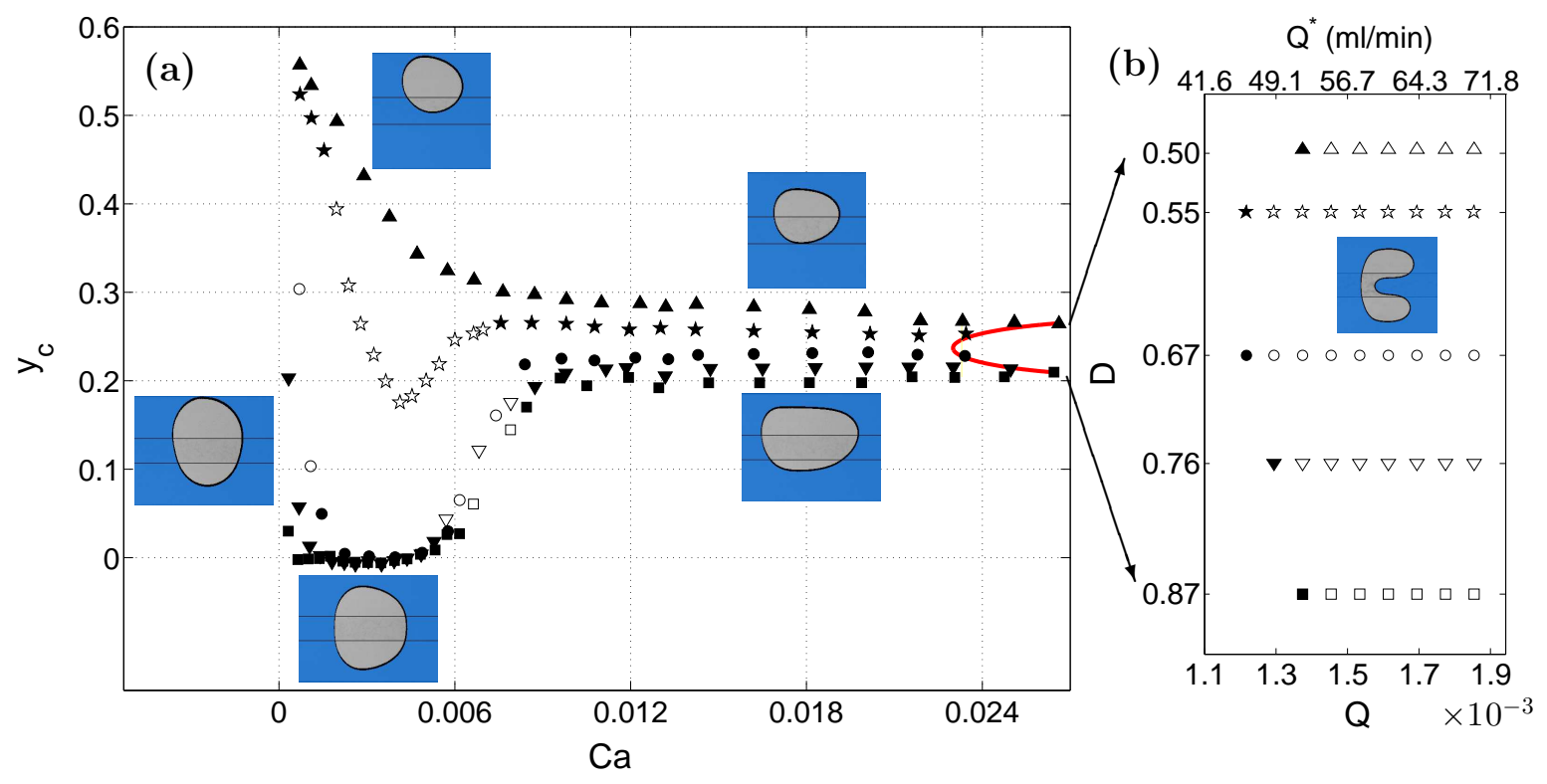

Figure 8. (a) Experimental centroid position of a steadily propagating bubble, started from a centred initial condition, as a function of capillary number for the same channel

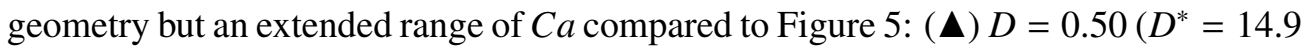
$\mathrm{mm}),(\star) 0.55(16.5 \mathrm{~mm}),(\bullet) 0.67(20.1 \mathrm{~mm}),(\boldsymbol{\nabla}) 0.76(22.8 \mathrm{~mm})$ and $(\boldsymbol{\square}) 0.87$ (26.1 $\mathrm{mm})$. The red line indicates the maximum values of $C a$ for which bubbles of different sizes propagate steadily. (b) Values of non-dimensional (dimensional) flow rates for which time-evolving dimpled bubbles are observed (open symbols) or remain convex (closed symbols) for five different bubble sizes.

system is bistable and the natural question now arises: which mode of propagation is selected at large $\mathrm{Ca}$ and does this change for different initial conditions?

Initially asymmetric bubbles driven with a large $C a$ always rapidly evolved towards a steadily propagating asymmetric configuration. However, when the bubble was initially centred, we found that the bubble did not converge onto a stable mode of propagation, but instead continually evolved in time until it broke up into two bubbles. This is illustrated in Figure 8 a, where the range of capillary numbers is extended compared to Figure 5. The red line in Figure 8 a denotes the largest value of $C a$ for which a steadily propagating asymmetric bubble is reached after starting from centred initial conditions; this boundary depends nonmonotonically on bubble size. For larger flow rates, as in Figure $8 \mathrm{p}$, the bubble moves into a double tipped configuration, and then breaks up. Note that the dimensionless imposed flow rate $Q=C a / U_{b}$ is used to characterise the unsteady bubbles instead of $C a$ because bubbles do not necessarily propagate with a constant speed.

The evolution of a bubble before and after break-up is illustrated in Figure 9, where its instantaneous shape and speed are shown as a function of time. When the bubble is set into 

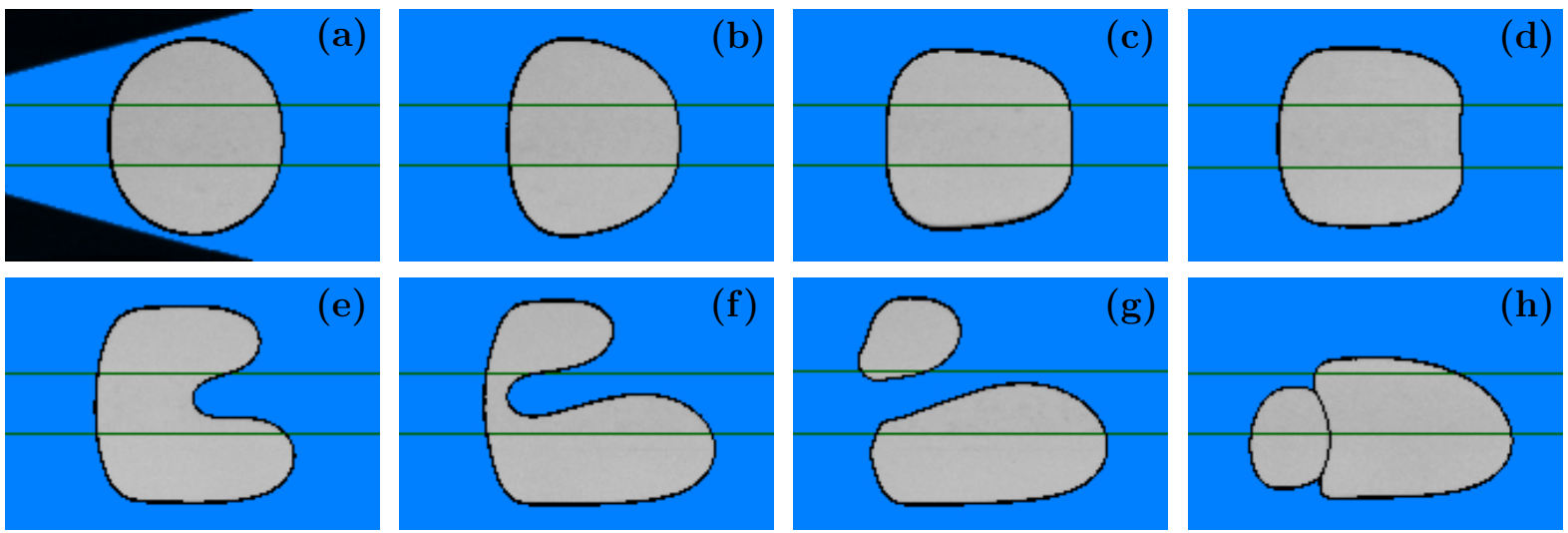

$$
\times 10^{-2}
$$

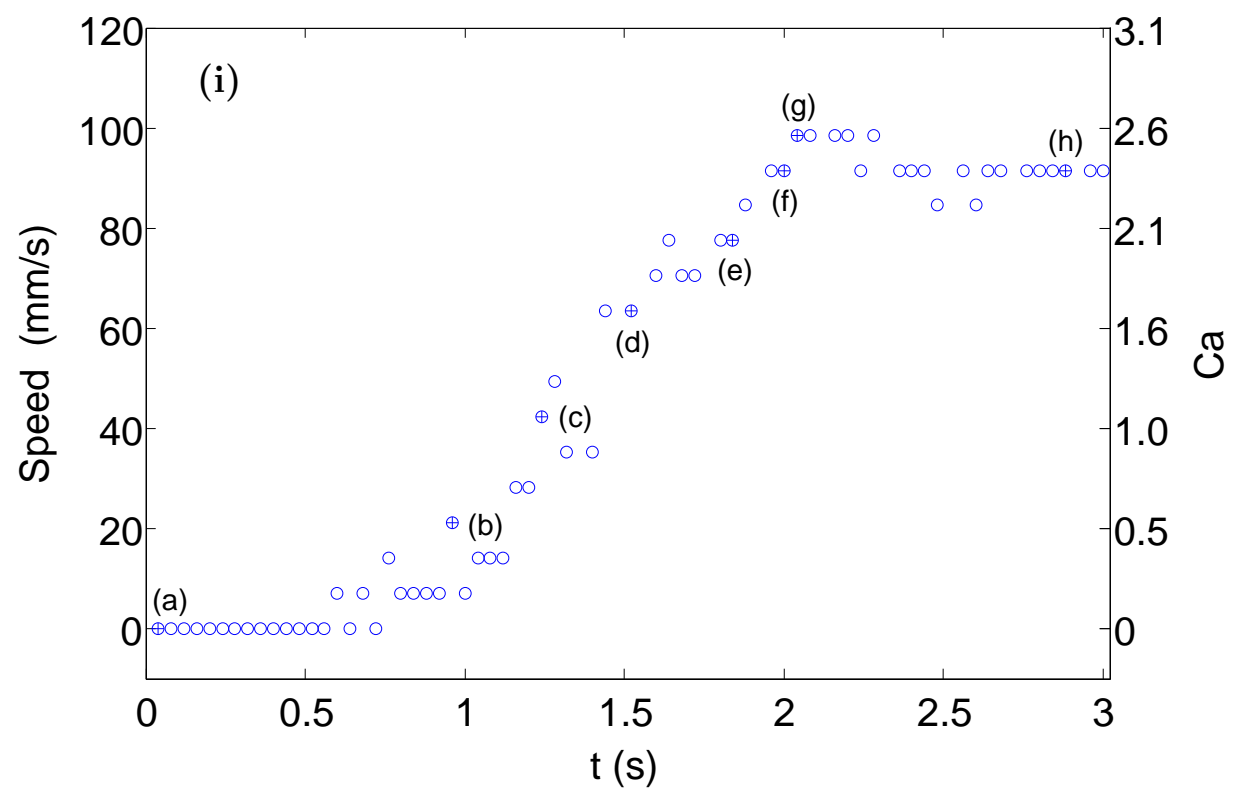

Figure 9. (a-h) Evolution of an unsteady bubble with diameter $D=0.77\left(D^{*}=23.15\right.$ $\mathrm{mm}$ ) propagating under a large flow rate $Q^{*}=100 \mathrm{ml} / \mathrm{min}$, from a centred initial condition. The channel geometry is the same as in Figure 5. The plot, which shows the instantaneous speed of the bubble tip $(\mathrm{Ca})$ as a function of time, with labels $(\mathrm{a}-\mathrm{h})$ referring to the snapshots, indicates that the bubble eventually reaches a steady state of changed topology.

motion from a centred position (a), it rapidly expands across the channel (b, c) and its front deforms over the rail to form an approximately centred dimple (d), because of the increased local viscous resistance above the centred rail. The bubble tips thus formed on either side of this dimple are displaced at a faster speed than the central region of the front $(e, f)$. Typically, one tip develops more rapidly than the other, so that the bubble evolves asymmetrically about the centreline of the channel. If the asymmetry remains moderate, the growth of the two 
tips results in the break-up of the bubble into two smaller bubbles (g), which aggregate to reach a steady mode of propagation (h). The evolution of the bubble is reflected in the plot of instantaneous speed as a function of time, which shows a monotonic increase towards a final steady propagation speed.

In terms of nonlinear dynamics, this means that the centred initial bubble does not lie within the basin of attraction of steady modes of propagation for sufficiently large imposed flow rates and its dynamics is affected by the presence of unstable solutions. The first RVB solution is weakly unstable in the sense that only two of the associated eigenvalues are unstable (repelling), based on a linear stability analysis performed when computing the results for Figure 7; the other eigenvalues are stable (attracting) and locally span a (highdimensional) space known as the stable manifold. Thus, once set into motion, we conjecture that the evolving bubble is transiently attracted towards the vicinity of the stable manifold of the first RVB solution described in section 4.3 (dimpled front).

As discussed in section 4.3 , the first RVB solution is part of an infinite countable family of bubbles solutions parametrised by the number of dimples exhibited by their front [18, 34]. Thus, our next question is: could the initially centred bubble transiently explore other unstable RVB states? This hypothesis was explored for a slightly larger channel aspect ratio $\alpha=40$ and rail width $w^{*}=10.0 \mathrm{~mm}$. The results are shown in Figure 10 in the parameter plane spanning bubble diameter and imposed flow rate. The fluid viscosity has been increased to enable larger dimensionless flow rates (up to 3.5 times larger than in Figure 8) while maintaining good visualisation given limitations on frame rate, and two of the three bubbles tested are smaller than previously shown. For moderate values of $Q$ the bubble propagates steadily in an asymmetric configuration. However, as $Q$ is increased, bubble configurations with one, two or three dimples are successively selected, which suggests that the bubble also transiently explores the second and third RVB solutions, before breaking-up to reach a topologically distinct steady mode of propagation.

\section{Summary and conclusion}

We have considered the propagation of individual gas bubbles within a Hele-Shaw channel containing a centred, axially-uniform constriction. We have considered the transition in behaviour as the bubble diameter is varied from the width of the rail to the width of the channel, thus bridging the two recent studies of Franco-Gómez et al. [23, 25]. For bubbles large enough that they span the entire channel width, the behaviour of the system is the same as the previously studied system of an air finger propagating into a similar channel geometry [22, 23]. The stable static bubble configuration is symmetric and as the flow rate and hence capillary number increases, the system undergoes a symmetry-breaking bifurcation at a critical capillary number $C a_{\mathrm{c} 2}$, above which the bubble propagates steadily in one of the deeper side channels on either side of the constriction. The local viscous resistance of 


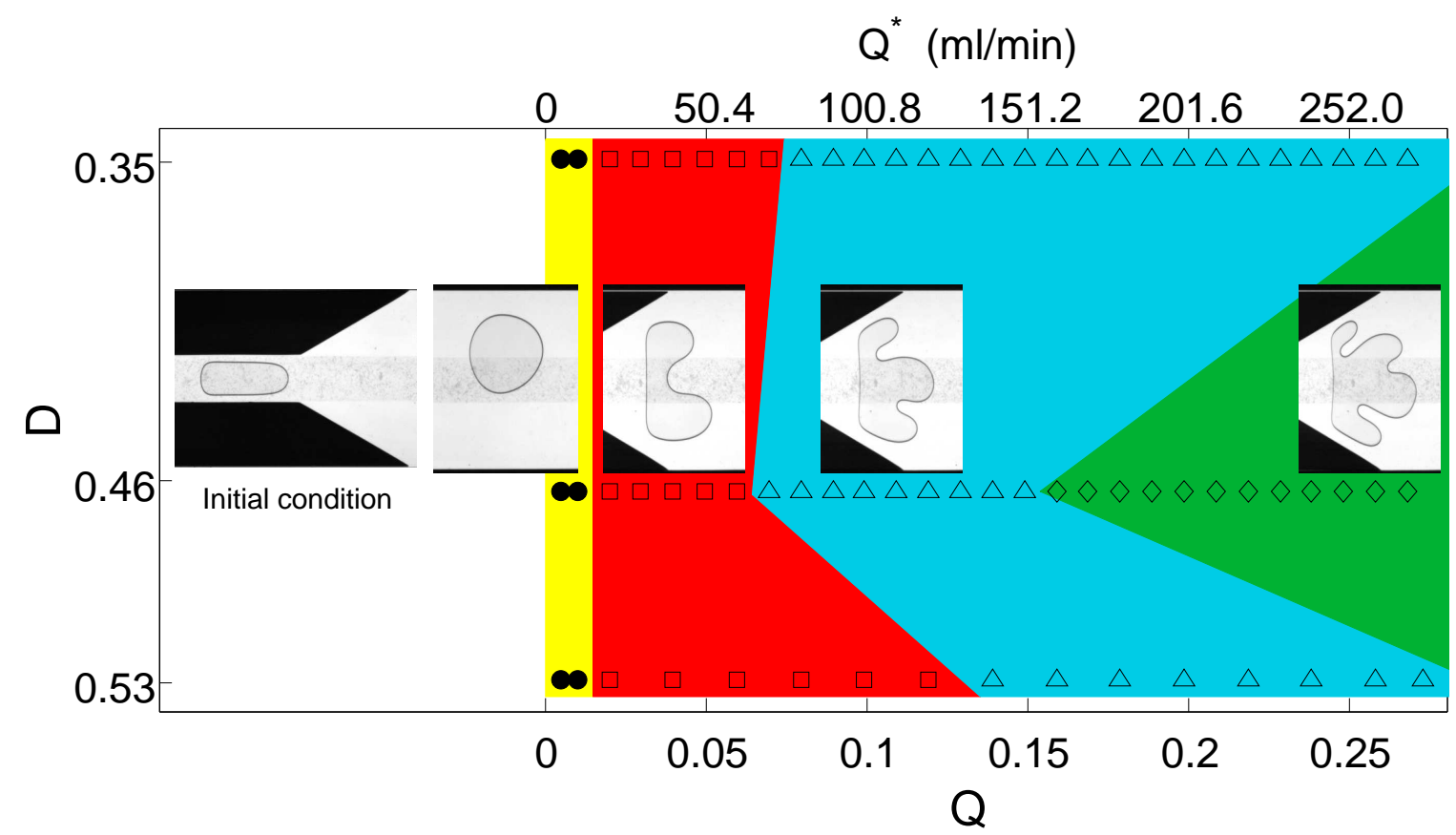

Figure 10. Experimental modes of propagation of an initially centred bubble in the parameter plane spanning bubble diameter and flow rate. Solid (open) symbols represent steady (unsteady) propagation: $(\bullet)$ steady asymmetric bubble; ( $\square$ ) doubletipped bubble, $(\triangle)$ triple-tipped bubble, $(\diamond)$ quadruple-tipped bubble. Note that the channel used for these experiment has an aspect ratio of $\alpha=40$, rail width $w=10.0 \pm 0.1 \mathrm{~mm}$, and height $h=24 \pm 1 \mu \mathrm{m}$, and that the silicone oil is more viscous than previously, with $\mu^{*}=5.0 \times 10^{-2} \mathrm{~Pa}$ s. The coloured regions are indicative only and used to distinguish different modes of steady (solid symbols) or unsteady (open symbols) modes of propagation.

the channel is non-uniform and the magnitude of the induced flow variations increases with capillary number, which underlies the symmetry-breaking bifurcation [22], beyond which it is more favourable to displace less fluid from the high-resistance, constricted region.

As the bubble size decreases it does not span the entire channel and the sidewalls can no longer stabilise the symmetric static solution. Hence, the static bubble occupies an asymmetric position. The introduction of axial flow can stabilise the symmetric solution through a symmetry-regaining pitchfork bifurcation at a critical capillary number $C a_{\mathrm{c} 1}<$ $C a_{\mathrm{c} 2}$. The stabilisation mechanism involves a subtle interplay between the bubble shape, surface tension and viscous forces and only operates when $C a_{\mathrm{c} 1}<C a<C a_{\mathrm{c} 2}$. We find that a depth-averaged model can accurately reproduce the bubble shapes for asymmetric and symmetric bubbles over the range of $C a$ studied. The bubble speeds are less accurately captured due at least in part to the presence of films above and below the bubble in the experiment, which are neglected in the model (see Appendix B). As Ca increases the projected 
areas of the experimental bubbles becomes greater than those of the model simulations because of the thickening of these fluid films. The percentage error in projected area increases approximately linearly with $C a$ and remains below $15 \%$ for all $C a$ studied. As the bubble size is further reduced, the two pitchfork bifurcations move closer together and eventually merge at a critical bubble volume. Below this volume, the bubble always propagates asymmetrically if $C a<0.023$, but at larger $C a$, the bubble may break-up during its transition to an asymmetric state.

We used numerical simulations to compute both steady and unsteady solutions and found that for higher $\mathrm{Ca}$ there are additional disconnected solution branches that correspond to the Romero-Vanden-Broeck solutions, originally discovered in air-finger flow [32, 33], but also found for finite bubbles in unbounded Hele-Shaw cells [17, 18]. The results indicate that the first of these solution branches is symmetric and initially unstable, but restabilises at sufficiently high capillary numbers. From the simulations, therefore, we expect the system to be bistable in this region with the choice of the final steady propagating state depending on the initial conditions. Experimentally, we find that when starting from asymmetric initial conditions the system rapidly approaches the asymmetric state. However, when starting from symmetric initial conditions the system exhibits complex dynamics in which it appears to transiently explore the weakly unstable isolated solution branches, resulting in double-, tripleand even quadruple-tipped bubbles. These unstable bubbles evolve ultimately leading to a topology change of the bubbles. This scenario, in which an increasing number of weakly unstable states develops as the flow rate increases, is reminiscent of the recent dynamical systems interpretation of the transition to turbulence in shear flows [26, 35]. Thus it is possible that a similar dynamic scenario occurs in the present system and provides an underlying structure and organisation to the complex phenomena of bubble break-up and topological change. We leave further pursuit of these ideas for future publications.

\section{Acknowledgements}

A.F.-G. was funded by CONICYT. This work was supported by the Leverhulme Trust (Grant RPG-2014-081).

\section{Appendix A. Sensitivity to the rail width and sharpness}

Profilometry measurements of the experimental rail reveal a geometry with approximately vertical sides, sharp edges and a rough upper surface. Averaged in the axial direction, the profile is well approximated by a rectangular shape (see supplementary material in [25]), and $\alpha_{w}=w^{*} / W^{*}$ is measured to be 0.233. For finger propagation, [23] found that taking $\alpha_{w}$ in (1) directly from the experimental profile gave quantitative agreement if $\alpha \geq 40$ and the sharpness parameter $s$ is fixed at 40. On the other hand, [25] studied bubble propagation in 

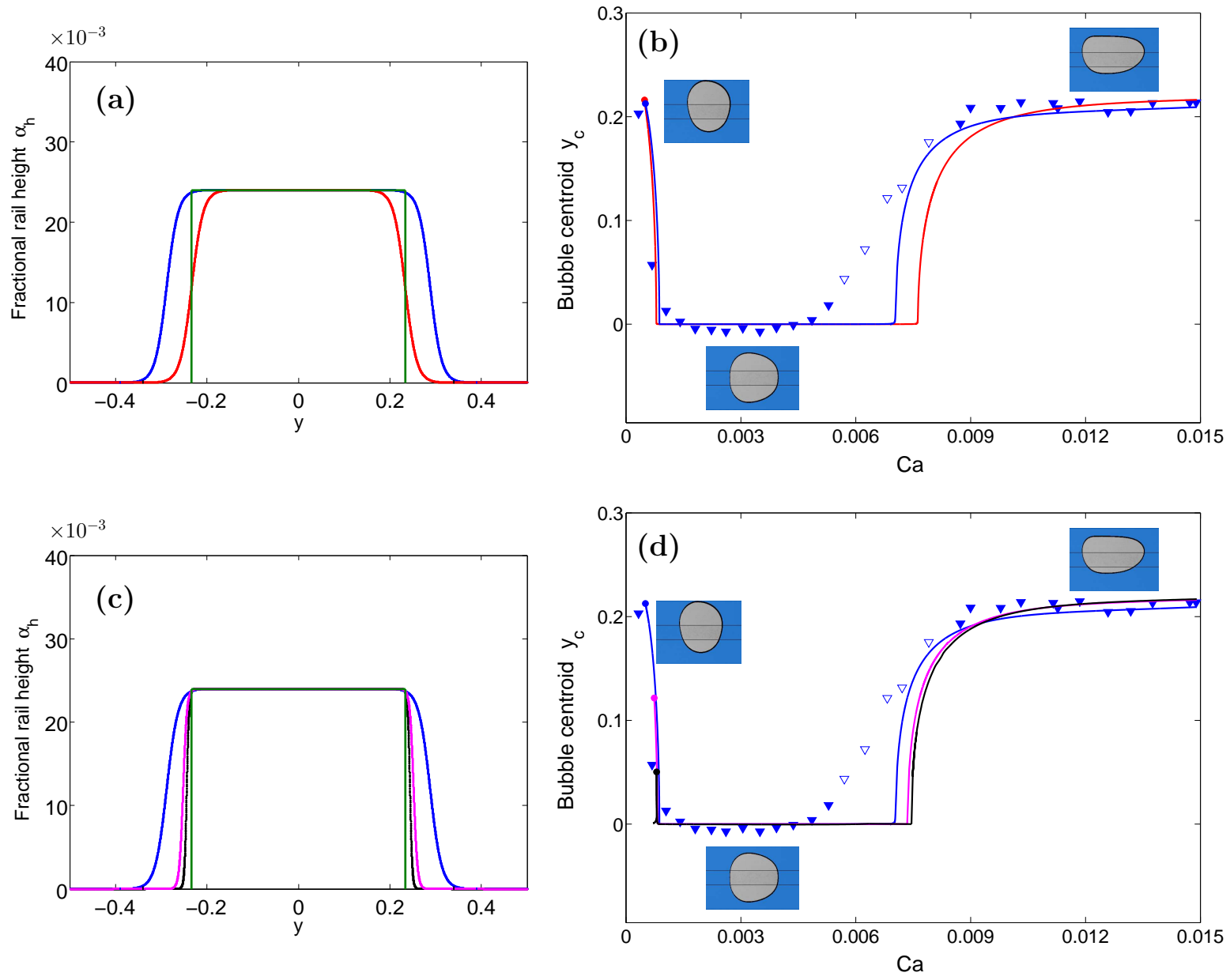

Figure A1. Test of numerical rail width and sharpness for the bifurcation diagram of a propagating bubble with diameter $D=0.76\left(D^{*}=22.8 \mathrm{~mm}\right)$. The symbols $(\boldsymbol{\nabla})$ in (b) and (d) correspond to the experimental results. The measured rail profile has $\alpha_{w}=0.233, \alpha_{h}=0.024$. For the numerical model, we test various rail parameters, each with $\alpha_{h}=0.024$ and $\alpha=30$ : (a,b) $\alpha_{w}=0.288, s=40$ (blue), $\alpha_{w}=0.233, s=40$ (red) and (c,d) $\alpha_{w}=0.252, s=120$ (magenta) and $\alpha_{w}=0.244, s=200$ (black)

the same channel geometry as in this paper, and found that better quantitative agreement is obtained by selecting $\alpha_{w}$ so that the width of the top-flat surface coincides with the width of the experimental rail; this process yields $\alpha_{w}=0.288$ if $s=40$. Figure A1a illustrates the rail profiles, with the solid green line corresponding to the experimental rail ( $\alpha_{w}=6.9 / 30=0.233$, with sharp vertical sides), and two smoothed model profiles: red line $\left(\alpha_{w}=0.233, s=40\right)$ and blue line $\left(\alpha_{w}=0.288, s=40\right)$.

In Figure $\mathrm{A} 1 \mathrm{~b}$, the experimental results for bubble centroid as a function of capillary number for a bubble of diameter $D=0.76\left(D^{*}=22.8 \mathrm{~mm}\right)$ are compared to the numerical results where $\alpha_{w}$ in (1) is taken to be either 0.288 and 0.233 . We find that using the wider rail 
profile in the model improves the agreement for the value of the second bifurcation point and the centroid values in the asymmetric region at larger $\mathrm{Ca}$, though there is very little difference between the results for the two rail widths at small $\mathrm{Ca}$.

The effect of sharpness is tested for $D=0.76$ using $s=40\left(\alpha_{w}=0.288\right), s=120$ ( $\left.\alpha_{w}=0.252\right)$ and $s=200\left(\alpha_{w}=0.244\right)$ and the rail profiles and corresponding bifurcations are displayed in Figure A1k, d, respectively. Each of these $s, \alpha_{w}$ pairs is chosen to match the width of the flat top of the rail profile. The sharper profiles give a more realistic representation of the physical rail, but in fact the bifurcation points for the smoothest rail (blue solid line) are the closest to the experimental results. Overall, we find the bifurcations of bubble propagation do not change significantly when increasing the rail sharpness. For the calculations in the body of this paper, we take $\alpha_{w}=0.288$ and $s=40$.

\section{Appendix B. Increase of bubble projected area with capillary number and estimate of thin film thickness}

Propagating bubbles of the diameters reported in Figure 5 were observed to increase their projected areas due to the dependence of film thickness on capillary number. The relative bubble area $A_{C a}^{*} / A_{0}^{*}$, where $A_{0}^{*}$ is the bubble area measured statically, is plotted as a function of the capillary number in Figure B1. For each bubble, there is a sudden increase of $5 \%$ in the initial range of $0 \leq C a \leq 3.26 \times 10^{-4}\left(0 \leq Q^{*} \leq 1 \mathrm{ml} / \mathrm{min}\right)$. There is followed by a slower increase of around $8 \%$ over the range $3.26 \times 10^{-4} \leq C a \leq 1.5 \times 10^{-2}\left(1 \leq Q^{*} \leq 30 \mathrm{ml} / \mathrm{min}\right)$. Over the whole range of $Q^{*}$, the increase of area from its static value is of the order of $13 \%$.

The normalised curves collapse inside a band of relative bubble area of approximately $\Delta\left(A_{C a}^{*} / A_{0}^{*}\right) \sim 0.015$, suggesting that the increase of fluid film thickness of the reported bubbles is independent of the bubble size within the investigated range of capillary numbers. Using the data in Figure B1, the average thickness of the thin films $t_{C a}^{*}$ are estimated with the geometrical method previously presented by [25] (supplementary material), $t_{C a}^{*}=\left(H^{*} / 2\right)\left(1-A_{0}^{*} / A_{C a}^{*}\right)$. At a capillary number of $C a=1.5 \times 10^{-2}\left(Q^{*}=30 \mathrm{ml} / \mathrm{min}\right)$, this estimate yields fluid films of average thickness $t_{C a}^{*} \sim 59 \mu \mathrm{m}$ with a variation of $\pm 5 \mu \mathrm{m}$ for the different bubble sizes. Note that this estimate is for the film thickness averaged over the whole bubble area, and so the film may be much thinner in places.

The quantitative comparisons shown earlier in this paper are for the model without any thin film corrections. We find that predictions of the bubble shape and centroid position are in good agreement with the numerical results. However, a comparison of dimensionless bubble speed $U_{b}$ vs $\mathrm{Ca}$ is in less good agreement (see Figure $\mathrm{B} 2 \mathrm{a}$ ), with the experimental bubble speed exceeding that predicted by the model by up to $15 \%$. This discrepancy is of the same order as the increase in bubble area, and shows a broadly similar dependence on $\mathrm{Ca}$.

As a first approximation to understand the effects of thin films, we assume that films occupy a spatially-constant fraction $\lambda$ of the channel height. In that case, the films 


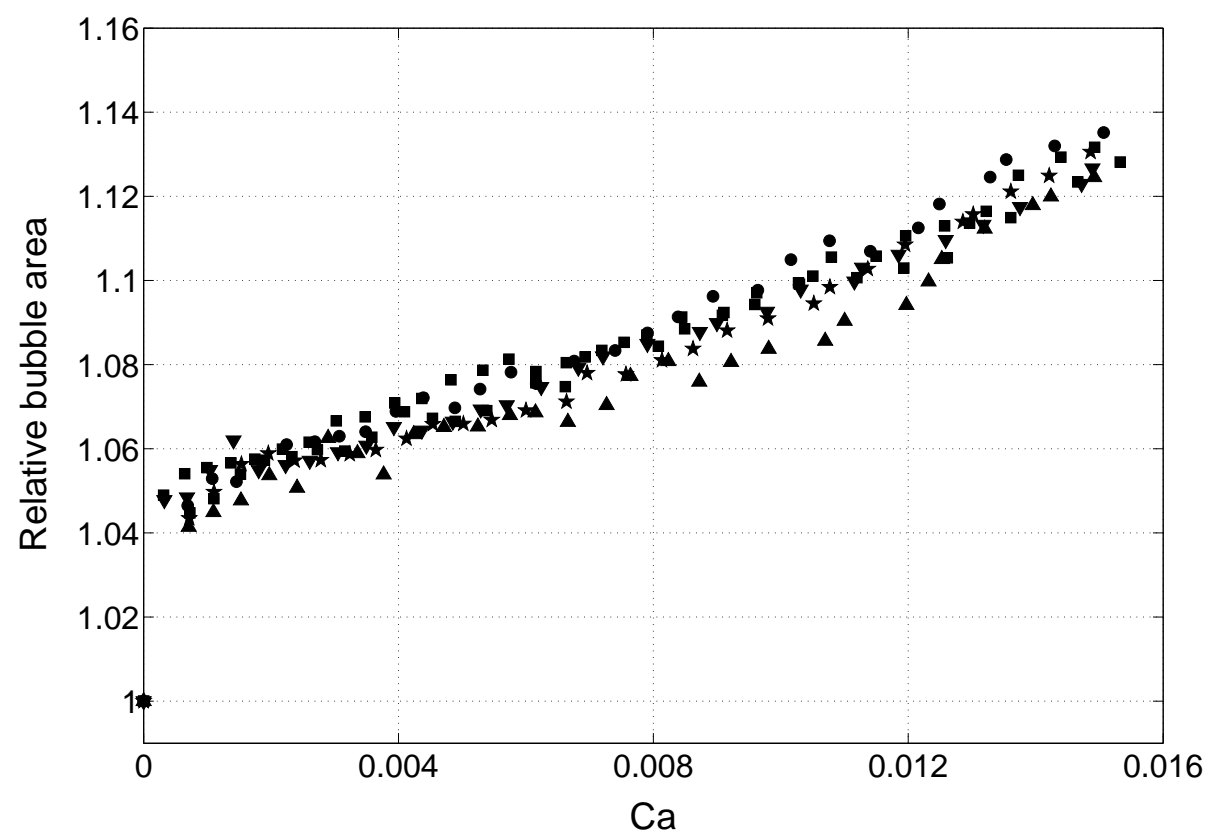

Figure B1. Relative bubble area $A_{C a}^{*} / A_{0}^{*}$, where $A_{0}^{*}$ is the bubble area measured statically, as a function of the capillary number $\mathrm{Ca}$; the initial condition is a centred static bubble. The plots reveal an increase of bubble area around $8 \%$ when the flow rate is increased from $C a=3.26 \times 10^{-4}(1 \mathrm{ml} / \mathrm{min})$ up to $C a=1.5 \times 10^{-2}\left(Q^{*}=30\right.$ $\mathrm{ml} / \mathrm{min})$. Note that there is a sudden increase of $5 \%$ between the static projected area $(C a=0)$ and the area measured at $C a=3.26 \times 10^{-4}(1 \mathrm{ml} / \mathrm{min})$. The change in projected area due to volume loss or gain along the length of the channel is better than $\pm 1 \%$. The symbols indicate different bubble sizes, using the same legend as in Figure 5 .

should appear in the kinematic boundary condition, the transverse curvature and the volume constraint. The latter two effects would in principle affect the bubble shape (which we know is in good agreement with the experiments), but the first would only lead to a rescaled $U_{b}$, as discussed by [15]. We do not have a predictive model for film thickness, so it is difficult to modify the model to match the experiments, but we can adjust the experimental results to match the dependence of the kinematic equation in the model by using the measurements of $A_{0}^{*} / A_{C a}^{*}$ as a proxy for $\lambda$. In the model, the bubble shape is determined by $\alpha$ and $Q$ and $C a$ is a derived parameter. We should, therefore, adjust both $U_{b}$ and $C a$ to compensate for the thin films, which leads to a closer agreement between experimental and model data (see Figure B2p).

\section{References}

[1] Baroud C N, Gallaire F and Dangla R 2010 Lab Chip. 10 2032-2045 

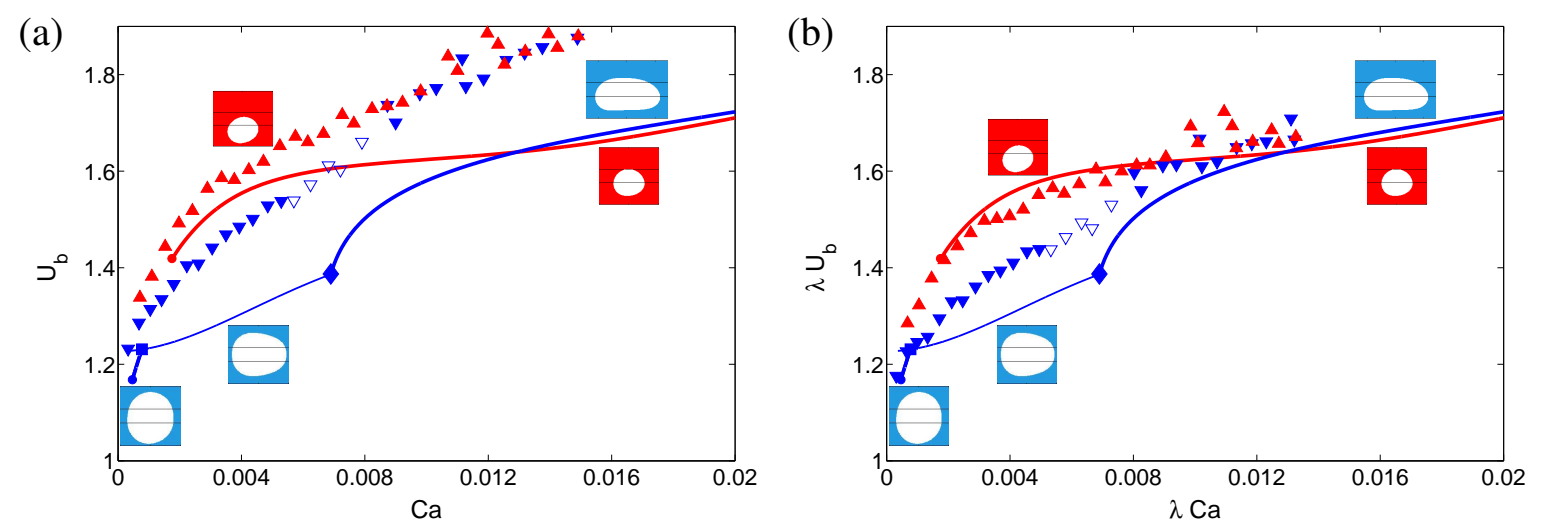

Figure B2. (a) Direct comparison of dimensionless propagation speed $U_{b}=C a / Q$ as a function of $C a$ for $D=0.50$ (red) and $D=0.76$ (blue) for original model and experiments. (b) The speed is adjusted to account for the presence of thin films by plotting $U_{b} \lambda$ against $C a \lambda$, where $\lambda$ is taken to be $A_{0}^{*} / A_{C a}^{*}$ for the experiments and $\lambda=1$ for the model; this correction yields a closer agreement between experiment and model than the uncorrected data, except for the case of the larger bubble at small flow rates. Here the discrepancy is likely to be due to the non-uniformity of the thin films in that regime.

[2] Stan C A, Guglielmini L, Ellerbee A K, Caviezel D, Stone H A and Whitesides G M 2011 Phys. Rev. E 84036302

[3] Sajeesh P and Sen A K 2014 Microfluid. Nanofluidics 17 1-52

[4] Dressler O J, i Solvas X C and deMello A J 2017 Annu. Rev. Anal. Chem. 10 1-24

[5] Di Carlo D, Irimia D, Tompkins R G and Toner M 2007 P Natl. Acad. Sci. USA 104 18892-18897

[6] Kim J, Erath J, Rodriguez A and Yang C 2014 Lab Chip 14 2480-2490

[7] Hashimoto M, Garstecki P and Whitesides G M 2007 Small 3 1792-1802

[8] Cox R G and Mason S G 1971 Ann. Rev. Fluid Mech 3 291-316

[9] Leal L G 1980 Ann. Rev. Fluid Mech 12 435-476

[10] Karnis A and Mason S G 1967 J. Colloid Interface Sci. 24 164-169

[11] Tanveer S and Saffman P G 1987 Phys. Fluids 30 2624-2635

[12] Abbyad P, Dangla R, Alexandrou A and Baroud C N 2011 Lab Chip. 11 813-821

[13] Yoon D H, Numakunai S, Nakahara A, Sekiguchi T and Shoji S 2014 RSC Adv. 4(71) 37721-37725

[14] Frot C, Taccoen N and Baroud C N 2016 PLoS One 11 1-12

[15] Saffman P G and Taylor G I 1958 Proc. R. Soc. Lond A 245 312-329

[16] Taylor G I and Saffman P G 1959 Q. J. Mech. Appl. Math. 12 265-279

[17] Tanveer S 1987 Phys. Fluids 30 651-658 
[18] Green C C, Lustri C J and McCue S W 2017 Proc. R. Soc. London A 47320170050

[19] de Lózar A, Heap A, Box F, Hazel A L and Juel A 2009 Phys. Fluids 21101702

[20] Pailha M, Hazel A L, Glendinning P A and Juel A 2012 Phys. Fluids 24021702

[21] Hazel A L, Pailha M, Cox S J and Juel A 2013 Phys. Fluids 25062106

[22] Thompson A B, Juel A and Hazel A L 2014 J. Fluid Mech. 746 123-164

[23] Franco-Gómez A, Thompson A B, Hazel A L and Juel A 2016 J. Fluid Mech. 794 343368

[24] Jisiou M, Dawson G, Thompson A B, Mohr S, Fielden P R, Hazel A L and Juel A 2014 Procedia IUTAM 11 81-88

[25] Franco-Gómez A, Thompson A B, Hazel A L and Juel A 2017 Soft Matter 138684 8697

[26] Kawahara G, Uhlmann M and van Veen L 2012 Annu. Rev. Fluid Mech. 44 203-225

[27] Barkley D 2016 J. Fluid Mech. 803 P1

[28] Reynolds O 1886 Phil. Trans. R. Soc. 177 157-234

[29] Heil M and Hazel A L 2006 Lecture Notes on Computational Science and Engineering 53

[30] Park C W and Homsy G M 1984 J. Fluid Mech. 139 291-308

[31] Reinelt D A and Saffman P G 1985 SIAM J. Sci. Stat. Comp. 6 542-561

[32] Romero L A 1982 PhD thesis, California Institute of Technology

[33] Vanden-Broeck J M 1983 Phys. Fluids 26 2033-2034

[34] Gardiner B P J, McCue S W and Moroney T J 2015 Results Phys. 5 103-104

[35] Gibson J F and Schneider T M 2016 J. Fluid Mech. 794 530-551 